\title{
A sample of radio galaxies spanning three decades in radio luminosity - I. The host-galaxy properties and black-hole masses
}

\author{
Ross J. McLure ${ }^{3,1 \star}$, Chris J. Willott ${ }^{3,2}$, Matt J. Jarvis ${ }^{5,3}$, Steve Rawlings ${ }^{3}$, \\ Gary J. Hill ${ }^{4}$, Ewan Mitchell ${ }^{3}$, James S. Dunlop ${ }^{1}$, Margrethe Wold ${ }^{6}$ \\ ${ }^{1}$ Institute for Astronomy, University of Edinburgh, Royal Observatory, Edinburgh, EH9 3HJ, UK \\ ${ }^{2}$ National Research Council, 5071 West Saanich Rd, Victoria, B.C., V9E 2E7, Canada \\ ${ }^{3}$ Astrophysics, Department of Physics, Keble Road, Oxford, OX1 3RH, U.K. \\ ${ }^{4}$ McDonald Observatory, University of Texas at Austin, RLM 15.308, Austin, TX 78712-1083, USA \\ च ${ }^{5}$ Sterrewacht Leiden, Postbus 9513, 2300 RA Leiden, The Netherlands \\ ${ }^{6}$ European Southern Observatory, Karl-Schwarzchild-Str. 2, D-85748 Garching, Germany
}

29 September 2006

\begin{abstract}
The results of analysis of HST $I$-band imaging of a sample of 41 radio galaxies spanning three orders of magnitude in radio luminosity at redshift $z \simeq 0.5$ are presented. These results represent the first stage in a coordinated programme to study the connection between radio luminosity and host-galaxy properties, black-hole masses and cluster environments in radio galaxies spanning a wide range in radio luminosity over a restricted range in redshift. The full sample is comprised of objects drawn from four complete, low-frequency selected radio samples with progressively fainter flux-density limits (3CRR, 6CE, 7CRS and the new TexOx-1000 sample). Modelling of the HST imaging data shows that the host galaxies have surface-brightness distributions consistent with those expected for classic ellipticals (Sérsic parameter, $\beta \simeq 0.25$ ), with $\beta$ in the range $0.17<\beta<0.30$, and a mean of $\langle\beta\rangle=0.23 \pm 0.01$. The luminosities of the host galaxies are found to be comparable with those of galaxies drawn from the bright end of the local cluster galaxy luminosity function, spanning the range $0.7 L^{\star}<L<10 L^{\star}$, with a mean of $3.2 \pm 0.3 L^{\star}$, after correcting for the effects of passive evolution. In addition, the radio galaxies are shown to follow a Kormendy $\left(\mu_{e}-r_{e}\right)$ relation indistinguishable from that of both powerful low-redshift radio galaxies and local Abell brightest cluster galaxies. Combining our new results with those in the literature it is found that the scalelengths and Kormendy relations of $3 \mathrm{C}$-class radio galaxies do not vary significantly over the redshift range $0.0<z<0.8$, providing no evidence for dynamical evolution of this class of host galaxy within this redshift interval. Converting the host-galaxy luminosities into black-hole mass estimates, using the local $M_{b h}-M_{b u l g e}$ correlation, predicts that the radio galaxies harbour central black holes with masses in the range $10^{8.1} \mathrm{M}_{\odot}<M_{b h}<10^{9.5} \mathrm{M}_{\odot}$, with a geometric mean of $\left\langle M_{b h}\right\rangle=10^{8.87 \pm 0.04} \mathrm{M}_{\odot}$. Finally, a significant $(\simeq 3 \sigma)$ correlation is found between black-hole mass and $151-\mathrm{MHz}$ radio luminosity for those objects in the sample with either high-excitation nuclear spectra (HEG) or classical double (CD) radio structures.
\end{abstract}

Key words: galaxies: active - galaxies: nuclei - galaxies: fundamental parameters

\section{INTRODUCTION}

The underlying mechanisms which govern the large range in low-frequency radio luminosity observed in radio-loud active galactic nuclei (AGN) are currently unknown. Over the

(C) 0000 RAS 
previous two decades a wealth of observational effort has been invested in studying the redshift régime $0<z<1$, investigating whether the dominant factors are linked to the Mpc-scale cluster environments (eg. Prestage \& Peacock 1988; Hill \& Lilly 1991; Ellingson, Yee \& Green 1991; Wold et al. 2000; Best 2000) or the kpc-scale properties of the host galaxies (e.g. Smith \& Heckman 1989; Best, Longair \& Röttgering 1998; McLure \& Dunlop 2000; Dunlop et al. 2003; Zirm, Dickinson \& Dey 2003).

One of the incontrovertible observational facts which has emerged from these studies, at least at $z<1$, is that the host galaxies of all powerful radio-loud AGN are massive $L>L^{\star}$ ellipticals (eg. Taylor et al. 1996; McLure et al. 1999; Dunlop et al. 2003). The apparent uniformity of radioloud AGN host galaxies has taken on added importance over the last few years, following the discovery in nearby (distance $\lesssim 150 \mathrm{Mpc}$ ) inactive galaxies that a reasonably accurate estimate $\left(\Delta M_{b h} \simeq 0.3 \mathrm{dex}\right)$ of the central blackhole mass can be obtained via its correlation with the mass of the host spheroidal component (Kormendy \& Richstone 1995; Magorrian et al. 1998; Gebhardt et al. 2000; Ferrarese \& Merritt 2000; McLure \& Dunlop 2002; Marconi \& Hunt 2003; Tremaine et al. 2002). Moreover, recent progress has also indicated that a similarly accurate black-hole mass estimate $\left(\Delta M_{b h} \simeq 0.4 \mathrm{dex}\right)$ can be obtained for broad-line AGN using emission-line widths to derive the virial mass estimate (eg. Kaspi et al. 2000; McLure \& Dunlop 2002; McLure \& Jarvis 2002; Vestergaard 2002). Consequently, a large body of work has appeared in the recent literature investigating the possible link between radio luminosity and black-hole mass in radio-loud AGN (eg. Laor 2000; Lacy et al. 2001; McLure \& Dunlop 2001a; McLure \& Dunlop 2002; Bettoni et al. 2003; Dunlop et al. 2003).

Unfortunately, observational studies have traditionally been subject to a degeneracy between radio luminosity and redshift produced as a by-product of flux-limited radio samples. In order to study the properties of radio-loud AGN separated by a large dynamic range in radio luminosity, it has previously been necessary to select samples consisting of objects covering a wide range of redshifts. This has led to difficulties in interpreting the data due to the complication of potentially significant evolutionary effects. However, by selecting our sample of objects from four complete, lowfrequency selected radio samples with successively fainter flux-density limits, it has been possible to construct a sample of radio galaxies which spans three decades in radio luminosity at a virtually constant cosmic epoch $(0.4<z<0.6)$. In this respect the motivation for this study is identical to that pursued by Hill \& Lilly (1991), who investigated the cluster environments of a sample of 45 radio galaxies spanning a similar range in radio luminosities at $z \simeq 0.5$. By successfully disentangling the effects of luminosity and redshift for the first time, Hill \& Lilly (1991) were able to demonstrate that $z \simeq 0.5$ marks an apparent epoch-dependent change in cluster environment, with radio galaxies tending to inhabit significantly richer cluster environments than their low-redshift counterparts. Furthermore, the wide range of radio luminosities in the Hill \& Lilly sample enabled them to determine that extended radio luminosity did not appear to be a strong function of cluster richness.

Developments over the intervening decade have provided our new study with two crucial advantages over the previous work of Hill \& Lilly (1991). Firstly, the availability of the 7CRS (Willott et al. 2003; Lacy et al. 1999) and TexOx-1000 (Hill \& Rawlings 2003) radio samples has now allowed us to compile a sample of 41 radio galaxies at $z \simeq 0.5$, which is complete over the full three-decades range in radio power. Secondly, with the high-resolution imaging provided by the Hubble Space Telescope (HST), and the improvement in follow-up observations available with groundbased telescopes, we are now in a position to obtain a wide range of high quality data-sets for our new sample which were not obtainable only ten years ago. Consequently, our new sample of $z \simeq 0.5$ radio galaxies is the basis for an ambitious project, which will for the first time systematically investigate the connection between the radio luminosity of radio galaxies, the properties of their host galaxies, their location on the fundamental plane, the richness and mass of their cluster environments and the masses of their central black holes.

The results presented in this paper represent the first stage of this wide-ranging project, and concentrate solely on the analysis of our deep $I$-band HST WFPC2 imaging data. The results of our extensive follow-up observations of the sample are deferred to a series of future papers.

The structure of the paper is as follows, in Section 2 the details of the radio-galaxy sample are described, while in Section 3 the HST observations and data reduction are discussed. In Section 4 the modelling of the HST imaging is described, with the main results presented and compared to literature results on low-redshift radio galaxies in Section 5. In Section 6 the host-galaxy properties of our 3CRR sub-sample are compared to literature results to explore the evidence for dynamical evolution of the most powerful radio galaxies within the redshift range $0.0<z<0.8$. In Section 7 the properties of the radio-galaxy hosts are compared to those of local brightest cluster galaxies. In Section 8 the masses of the radio galaxies' central black holes are estimated, and in Section 9 the relationship between black-hole mass and radio luminosity is investigated. The main conclusions are summarized in Section 10. Unless otherwise stated, throughout this paper the following cosmology is assumed: $H_{0}=70 \mathrm{~km} \mathrm{~s}^{-1} \mathrm{Mpc}^{-1}, \Omega_{m}=0.3, \Omega_{\Lambda}=0.7$.

\section{THE SAMPLE}

The full 41-object $z \simeq 0.5$ radio-galaxy sample (hereafter the ZP5 sample) consists of all the narrow-line radio galaxies in the redshift interval $0.4<z<0.6$ from four, complete, low-frequency selected radio surveys; 3CRR (Laing, Riley \& Longair 1983), 6CE (Eales et al. 1997; Rawlings, Eales \& Lacy 2001), 7CRS (Willott et al. 2003; Lacy et al. 1999) and TexOx-1000; hereafter TOOT (Hill \& Rawlings 2003). The locations of all four radio surveys on the radio luminosity - redshift plane are shown in Fig 1. Details of the ZP5 sample can be found in Table 1. Additionally, Table 2 lists those objects which satisfied the redshift and radio luminosity criteria for the ZP5 sample, but were excluded due to their broad-emission line spectra.

The TOOT objects were selected from a preliminary version of the survey. They are therefore a subset of all the TOOT sources meeting the radio flux density and sky area selection criteria. Broad-line objects were excluded, in the 
Table 1. The full ZP5 sample. Column one lists the radio-galaxy names, while columns two and three list the J2000 source coordinates. Column 4 lists the radio-galaxy redshifts and column five lists the logarithm of the 151-MHz luminosities in units of $\mathrm{WHz}^{-1} \mathrm{sr}^{-1}$. Column 6 classifies the radio structures as either Classical Double (CD), Fat Double (FD), Jet (J) or Compact (CM), the same classifications as used by Owen \& Laing (1989). The radio structure classifications are based on consistent $1.4 \mathrm{GHz}$ VLA A-array data (1.5 arcsec resolution) for the $6 \mathrm{CE}, 7 \mathrm{CRS}$ and TOOT sub-samples. For the 3CRR sub-sample, for which structural classifications are well established, the classifications were based on the high-quality maps available in the literature (at a variety of frequencies/resolutions). Column 7 describes the optical nuclear spectra in terms of the presence of high-excitation narrow emission lines (HEG) or not (LEG), using the classification scheme of Jackson \& Rawlings (1997). Those objects for which the available optical spectra are of insufficient quality to allow a reliable HEG/LEG determination are listed with a '?'. To avoid confusion we include here in parenthesis the old names of the 7CRS objects which have been adopted in previous papers: 7C0213+3418 (5C6.63), 7C0219+3419 (5C6.201), 7C0219+3423 (5C6.214), $7 \mathrm{C} 0220+2952$ (5C6.233), 7C0223+3415 (5C6.279), 7C0810+2650 (5C7.7).

\begin{tabular}{|c|c|c|c|c|c|c|}
\hline Source & RA & DEC & $\mathrm{z}$ & $L_{151}$ & Radio Structure & Class \\
\hline $3 \mathrm{C} 16$ & 003745.39 & +132009.6 & 0.405 & 26.82 & $\mathrm{CD}$ & HEG \\
\hline 3C 19 & 004055.01 & +331007.3 & 0.482 & 26.96 & $\mathrm{CD}$ & LEG \\
\hline $3 \mathrm{C} 46$ & 013528.47 & +375405.7 & 0.437 & 26.84 & $\mathrm{CD}$ & HEG \\
\hline 3C 172 & 070208.32 & +251353.9 & 0.519 & 27.17 & $\mathrm{CD}$ & HEG \\
\hline $3 \mathrm{C} 200$ & 082725.38 & +291845.5 & 0.458 & 26.92 & $\mathrm{CD}$ & LEG \\
\hline $3 \mathrm{C} 225 \mathrm{~B}$ & $09 \quad 42 \quad 15.41$ & +134551.0 & 0.582 & 27.50 & $\mathrm{CD}$ & HEG? \\
\hline $3 \mathrm{C} 228$ & 095010.79 & +142000.9 & 0.552 & 27.37 & CD & $\mathrm{HEG}$ \\
\hline 4C 74.16 & $1014 \quad 14.84$ & +743737.4 & 0.568 & 27.16 & $\mathrm{CD}$ & HEG \\
\hline $3 \mathrm{C} 244.1$ & 103333.97 & +581435.8 & 0.428 & 27.10 & $\mathrm{CD}$ & HEG \\
\hline $3 \mathrm{C} 274.1$ & 123526.64 & +212034.7 & 0.422 & 27.02 & $\mathrm{CD}$ & HEG \\
\hline $3 \mathrm{C} 295$ & 141120.65 & +521209.0 & 0.464 & 27.68 & $\mathrm{CD}$ & HEG \\
\hline $3 \mathrm{C} 330$ & 160935.01 & +655637.7 & 0.550 & 27.43 & $\mathrm{CD}$ & HEG \\
\hline 3C 341 & 162804.04 & +274139.3 & 0.448 & 26.88 & $\mathrm{CD}$ & HEG \\
\hline $3 \mathrm{C} 427.1$ & 210407.07 & +763310.8 & 0.572 & 27.53 & $\mathrm{CD}$ & LEG \\
\hline 3C 457 & $23 \quad 1207.57$ & +184541.4 & 0.428 & 27.00 & $\mathrm{CD}$ & HEG \\
\hline $6 \mathrm{C} 0825+3407$ & $08 \quad 2514.59$ & +340716.8 & 0.406 & 26.09 & FD & LEG \\
\hline $6 \mathrm{C} 0850+3747$ & 085024.77 & $\begin{array}{l}+374709.1\end{array}$ & 0.407 & 26.15 & FD & HEG \\
\hline $6 \mathrm{C} 0857+3945$ & 085743.56 & +394529.0 & 0.528 & 26.34 & $\mathrm{CD}$ & HEG \\
\hline $6 \mathrm{C} 1111+3940$ & 111119.39 & +394014.5 & 0.590 & 26.33 & $\mathrm{CD}$ & LEG \\
\hline $6 \mathrm{C} 1132+3439$ & 113245.74 & +343936.2 & 0.512 & 26.33 & $\mathrm{CD}$ & HEG \\
\hline $6 \mathrm{C} 1200+3416$ & 120053.34 & +341647.3 & 0.530 & 26.17 & $\mathrm{CD}$ & LEG \\
\hline $6 \mathrm{C} 1303+3756$ & 130344.26 & +375615.2 & 0.470 & 26.29 & $\mathrm{CD}$ & HEG \\
\hline $7 \mathrm{C} 0213+3418$ & $02 \quad 1328.39$ & +341830.6 & 0.465 & 25.66 & $\mathrm{CD}$ & LEG \\
\hline $7 \mathrm{C} 0219+3419$ & $\begin{array}{llll}02 & 19 & 15.89\end{array}$ & +341943.2 & 0.595 & 26.13 & FD & HEG? \\
\hline $7 \mathrm{C} 0219+3423$ & 021937.83 & +342311.2 & 0.595 & 25.98 & $\mathrm{~J}$ & HEG? \\
\hline $7 \mathrm{C} 0220+2952$ & 022034.26 & +295219.5 & 0.560 & 26.07 & FD & LEG? \\
\hline $7 \mathrm{C} 0223+3415$ & 022347.24 & +341511.9 & 0.473 & 25.55 & $\mathrm{CD}$ & HEG \\
\hline $7 \mathrm{C} 0810+2650$ & $\begin{array}{lll}08 & 10 & 26.10\end{array}$ & $\begin{array}{r}+265049.1 \\
\end{array}$ & 0.435 & 25.58 & $\mathrm{CM}$ & HEG \\
\hline $7 \mathrm{C} 1731+6638$ & 173143.84 & +663856.7 & 0.562 & 25.62 & $\mathrm{CD}$ & HEG \\
\hline $7 \mathrm{C} 1806+6831$ & 180650.16 & +683141.9 & 0.580 & 26.36 & $\mathrm{CD}$ & $\mathrm{HEG}$ \\
\hline TOOT $0009+3523$ & 000946.90 & +352345.1 & 0.439 & 24.79 & FD & LEG \\
\hline TOOT $0013+3459$ & 001313.29 & +345940.6 & 0.577 & 25.75 & FD & LEG \\
\hline TOOT $0018+3510$ & 001853.93 & +351012.1 & 0.416 & 25.16 & $\mathrm{~J}$ & LEG \\
\hline TOOT $1255+3556$ & 125555.83 & +355635.8 & 0.471 & 25.01 & $\mathrm{~J}$ & LEG \\
\hline TOOT $1301+3658$ & 130125.03 & +365809.4 & 0.424 & 24.76 & $\mathrm{~J}$ & LEG \\
\hline TOOT $1303+3334$ & 130310.29 & +333407.0 & 0.565 & 25.66 & $\mathrm{~J}$ & HEG \\
\hline TOOT $1307+3639$ & 130727.07 & +363916.4 & 0.583 & 25.30 & $\mathrm{~J}$ & LEG \\
\hline TOOT $1309+3359$ & 130953.95 & +335928.2 & 0.464 & 24.91 & $\mathrm{CM}$ & HEG \\
\hline TOOT $1626+4523$ & 162648.50 & +452342.6 & 0.458 & 25.03 & FD & LEG \\
\hline TOOT $1630+4534$ & 163032.80 & $\begin{array}{r}+453426.0 \\
\end{array}$ & 0.493 & 25.17 & $\mathrm{~J}$ & LEG \\
\hline TOOT $1648+5040$ & 164826.19 & +504058.0 & 0.420 & 25.12 & $\mathrm{CM}$ & LEG \\
\hline
\end{tabular}

case of TOOT $1252+3310$ after HST imaging and during further spectroscopic follow-up. There is some possibility that this subset is biased in the sense that the optically faintest sources may be have been preferentially excluded, although the level of bias will only be clear once analysis of the full survey is complete.

The original decision to select the radio-galaxy sample in the redshift interval $0.4<z<0.6$ was motivated by several factors. Principal among these was that at $z \simeq 0.5$ it becomes possible to get a full three decades of dynamic range in radio luminosity, including objects which are close to the most radio luminous objects which exist. Secondly, at $z \simeq 0.5$ it is possible to construct sub-samples, divided by radio luminosity, which contain sufficient numbers of objects to 


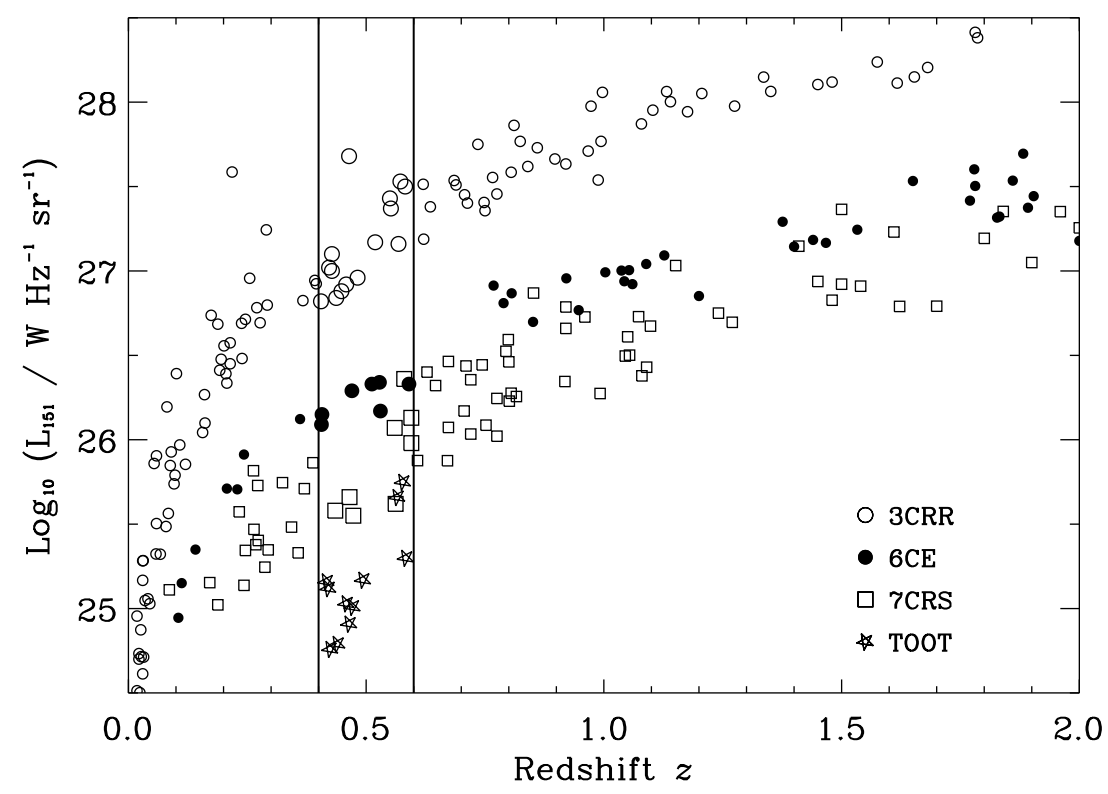

Figure 1. 151-MHz radio luminosity versus redshift for the narrow-line radio galaxy component of the $3 \mathrm{CRR}, 6 \mathrm{CE}$ and $7 \mathrm{CRS}$ samples. The members of the ZP5 sample are highlighted as enlarged symbols in the redshift interval $0.4<z<0.6$, including those drawn from the new TexOx-1000 (TOOT) survey.

Table 2. List of sources which met the redshift and radio-luminosity criteria for inclusion in the ZP5 sample, but were excluded due to the presence of broad emission lines. In column 2 the sources are classified as either quasar (Q) or weak quasar (WQ) according to the classification scheme of Jackson \& Rawlings (1997). We note here that the excluded quasars are confined to the two most radio luminous sub-samples, in agreement with the findings of Willott et al. (2000) that the quasar fraction in complete, low-frequency selected radio samples is an increasing function of radio luminosity. The implications of the possible quasar cores in the ZP5 sample (see Table 3) will be fully discussed in a forthcoming paper (Mitchell et al., in prep).

\begin{tabular}{lcl}
\hline Source & Class & Comment \\
\hline 3C 47 & $\mathrm{Q}$ & \\
3C 147 & $\mathrm{Q}$ & \\
3C 215 & $\mathrm{Q}$ & \\
3C 275.1 & $\mathrm{Q}$ & \\
3C 334 & $\mathrm{Q}$ & \\
3C 455 & $\mathrm{QQ}$ & \\
6C $1220+3723$ & $\mathrm{Q}$ & B1950.0 name - as in Rawlings et al. (2001) \\
5C 7.118 & WQ & B1950.0 name - as in Willott et al. (2003) \\
TOOT $1252+3310$ & WQ & Broad H $\beta$ detected in follow-up spectroscopy (Willott et al., in prep) \\
\hline
\end{tabular}

reliably determine the mean values and variances of relevant parameters, allowing any correlations with radio luminosity to be investigated. Thirdly, the chosen redshift range makes it possible to obtain the deep, high-resolution images necessary to derive accurate host-galaxy parameters, without requiring prohibitive amounts of $\mathrm{HST}$ time, while the upper limit of $z=0.6$ ensures that with the WFPC2 filters we are still able to sample light longward of the $4000 \AA$ break. Finally, redshift $z \simeq 0.6$ represents the maximum redshift for which it is practicable on $4 \mathrm{~m}$-class telescopes to obtain accurate measurements of the host-galaxy stellar-velocity dispersions, necessary to investigate the radio-galaxy fundamental plane properties (Willott et al., in prep).

\section{OBSERVATIONS AND DATA REDUCTION}

The observations for 39/41 of the objects in this project were carried out in the Cycle 10 program GO\#9045 with the Hubble Space Telescope. We used the WFPC2 detector with the F785LP filter. When combined with the system efficiency this filter has a peak wavelength of $8700 \AA$ and high throughput from $7900 \AA-9300 \AA$. This filter was selected because we wish to be sampling light above the $4000 \AA$ break, where there is less likely to be contamination of the old-stellar population by more recently formed stars or AGN-related emission. Further restrictions on the filter choice came from our desire to be free from emission lines as much as possible. The [O III] $\lambda 5007$ line only enters the filter bandpass at redshifts $z>0.58$ and $\mathrm{H} \alpha$ leaves the filter 
bandpass at redshifts $z>0.42$. Since our sample spans the redshift range $0.4<z<0.6$ this ensures very few sources will have significant emission-line contamination with this filter.

The radio galaxies were placed in the WF3 chip so that information on their arcminute-scale environments could be probed with the other chips on WFPC2. The orientation of the detector on the sky was arranged so that diffraction spikes and blooming from bright stars did not affect the array at the locations of the radio galaxies. The observations of each radio galaxy were made during a single orbit and the total exposure time of 2000 seconds was split into 4 integrations of 500 s each to facilitate the removal of cosmic rays. The target was dithered $5.5 \mathrm{WF} 3$ pixels in $x$ and $y$ in between integrations to improve the sampling of the PSF.

Calibrated images were retrieved from the HST archive. Registering and combining the 4 images per target were performed using the DRIZZLE routines in the IRAF package (Fruchter \& Hook 2002). The final reduced images for all four CCDs were sampled on a grid of pixels with size 0.0498 arcsec. Unsaturated stars typically have FWHM values of 3 pixels corresponding to 0.15 arcsec.

Existing observations were used in the analysis of the two remaining objects in the sample; $3 \mathrm{C} 16$ and $3 \mathrm{C} 295$. In the case of 3C16, an existing HST WFPC2 observation using the PC chip and the F702W filter was obtained from the HST archive (GO\#6675). The pixel size of the PC chip (0.045 arcsec/pixel) is well matched to that of the final drizzled images of the rest of the sample, and because $3 \mathrm{C} 16$ is at the low-redshift end of the sample $(z=0.405)$, the F702W filter is still sufficiently red to sample light longward of the $4000 \AA$ break. Although an archival HST observation of 3C295 was also available, because of the placement of the radio galaxy close to the edge of the PC chip, it was deemed unsuitable for the host-galaxy analysis described in Section 4. Fortunately we were provided with a deep $I$-band image of the 3C295 cluster obtained on the $2.5 \mathrm{~m}$ Nordic Optical Telescope for the weak lensing analysis of Wold et al. (2002). The final image comprises 10,000 seconds of integration and has a FWHM of 0.8 arcsecs. Although this is clearly lower spatial resolution than the HST data, this does not have any influence on the accurate determination of the host-galaxy properties because 3C295 is the largest, most luminous target in the whole sample.

\section{HOST-GALAXY ANALYSIS}

Given the high resolution and depth of the HST data available for this study it was decided that the determination of the host-galaxy parameters should be carried out via full two-dimensional modelling of the images. The twodimensional modelling technique adopted is identical to that which has previously been applied in the analysis of both radio galaxies and quasar host galaxies (eg. McLure et al. 1999; McLure \& Dunlop 2000; Dunlop et al. 2003). Full details of this modelling technique have been previously published in McLure, Dunlop \& Kukula (2000) and so only a brief outline will be provided here.

The first stage in the process is the production of a two-dimensional model host galaxy which has a surface- brightness distribution governed by an axially-symmetric Sérsic (1968) profile of the form:

$\Sigma(r)=\Sigma_{0} \exp \left[-\left(\frac{r}{r_{0}}\right)^{\beta}\right]$

where $\Sigma_{0}$ is the central surface brightness, $r_{0}$ is the characteristic scalelength and the $\beta$ parameter controls the shape of the radial profile. Under the adopted form of the Sérsic profile a value of $\beta=0.25$ corresponds to a classical de Vaucouleurs $r^{1 / 4}$ law (de Vaucouleurs 1953) and a value of $\beta=1.0$ corresponds to an exponential Freeman (1970) disc model. This model galaxy is subsequently convolved with the HST point spread function in order to produce a synthetic host-galaxy image. A chi-square comparison of the data and model is then performed on a pixel-by-pixel basis, in order to determine the goodness of fit of the initial model. A chi-square minimization process is then performed to determine the best-fitting value of the six parameters which describe the model $\left(\Sigma_{0}, r_{0}, \beta\right.$, axial ratio, position angle and nuclear point-source luminosity). Although the model galaxy axial ratio and position angle are included as free parameters during the fitting process, they are held constant as a function of radius. During the model fitting process it was not assumed a priori that the radio galaxies would not possess an unresolved nuclear point source.

\subsection{Point spread function}

For the analysis of the HST imaging data we utilized the model WFPC2 point spread functions (PSF) produced by the TinyTim software package (TinyTim v6.0, Krist 2001). The great advantage of using the TinyTim model PSFs over empirical WFPC2 PSFs is that they have infinite signalto-noise, and can be produced to match the $(x, y)$ coordinates of each radio galaxy on the WF3 chip. Although the model PSFs produced by TinyTim do not reproduce the large-scale halo of scattered light of the empirical WFPC2 PSF at radii of $r>1.5^{\prime \prime}$, this was not considered to be a serious problem for the current analysis because the luminosity of the any nuclear point sources were anticipated to be only a small fraction of the host-galaxy luminosity. This assumption proved to be justified, with the vast majority of the radio galaxies proving to possess a negligible nuclear point-source component (the median nuclear:host flux ratio for the ZP5 sample is 0.03).

In order to match the final reduced radio-galaxy images as accurately as possible, model PSFs were produced at the two dithered positions of each radio-galaxy observation separately. The two model PSFs were then processed by the DRIZZLE routine in an identical fashion to the radiogalaxy images to produce the final model PSF used during the modelling process.

\section{RESULTS}

In this section the results of the two-dimensional modelling of the host galaxies of the ZP5 sample are presented and discussed. A list of the principal host-galaxy parameters derived from the model fits for each individual object can be found in Table 3, while the average values for parameters of particular interest for the four radio-galaxy sub-samples 


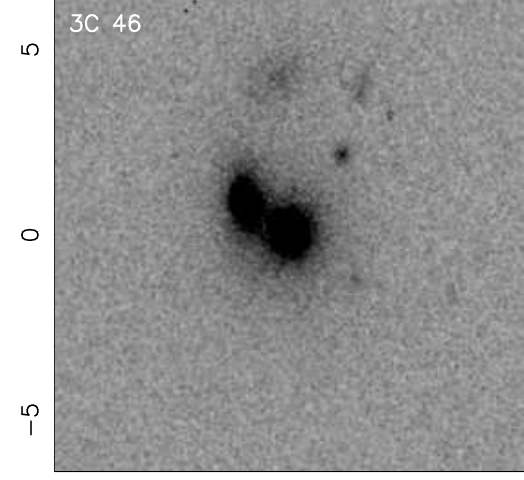

$-5$

0

ம

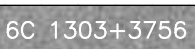

o

1

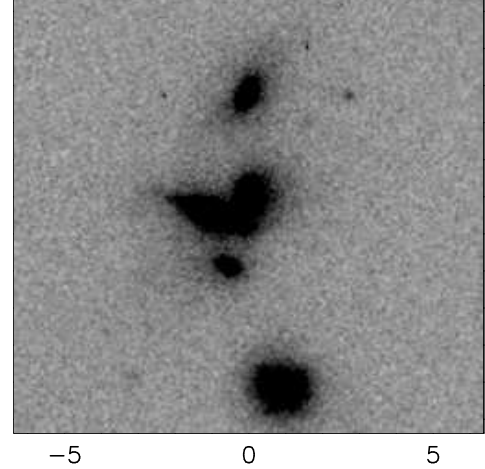

เก

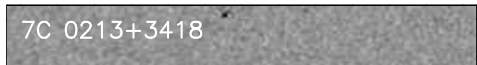

0

?
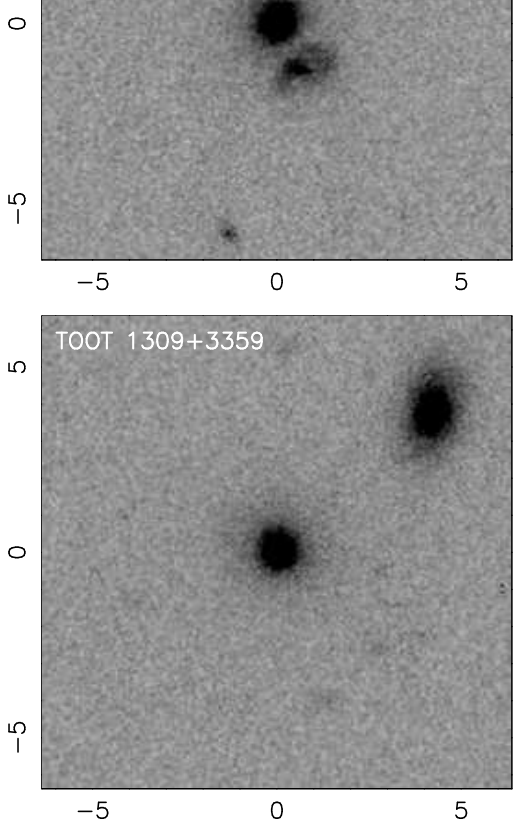

$\infty$

o

1

$-5$

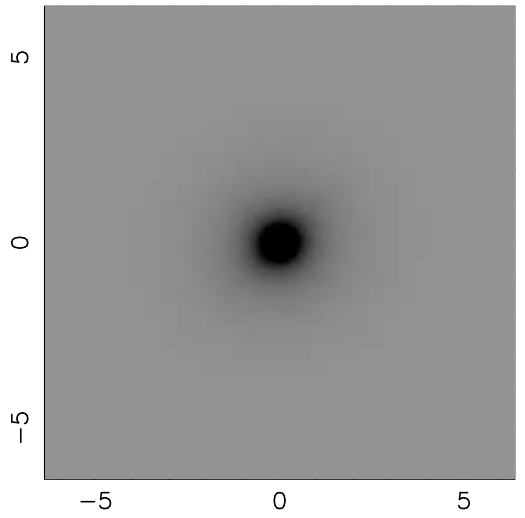

เ
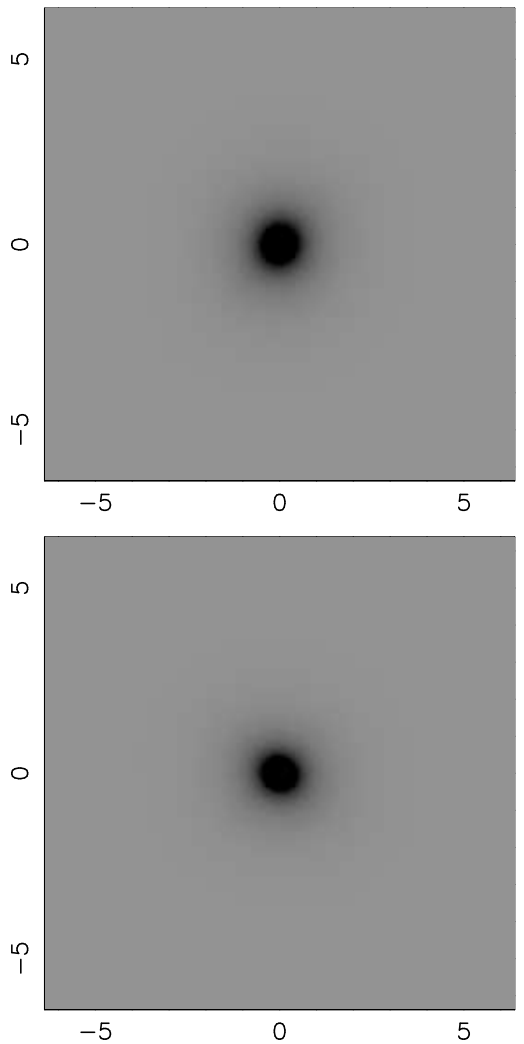

เ

0

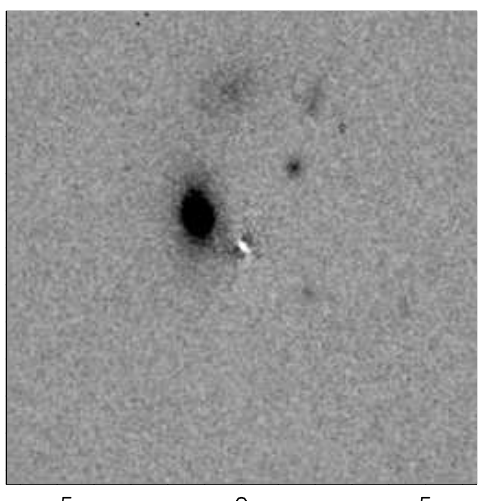

ก

0

เ

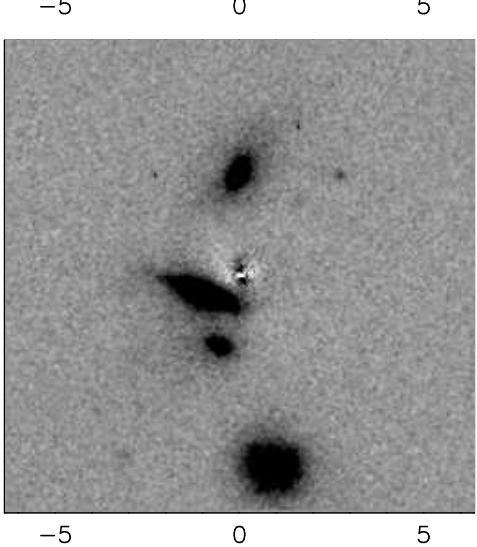

م

o

เ
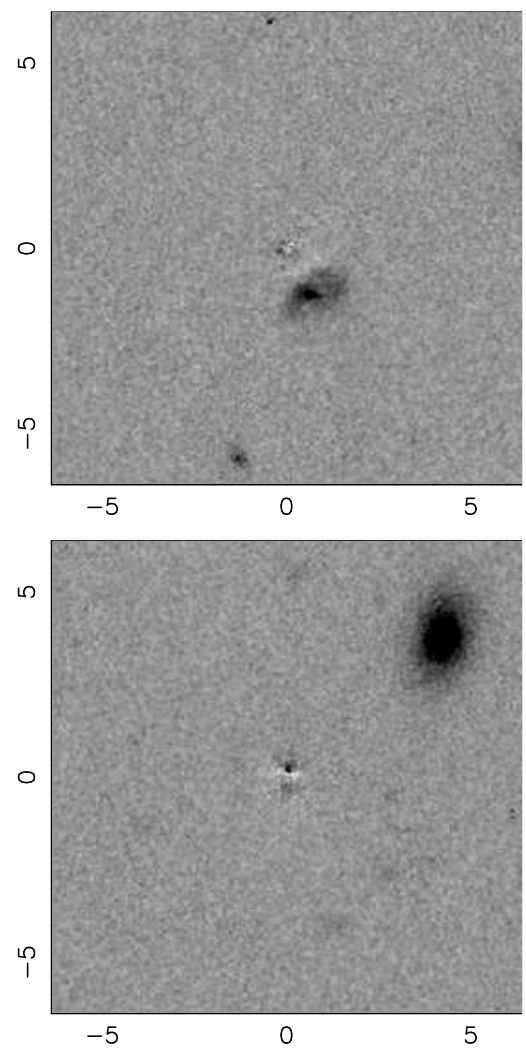

Figure 2. Examples of the two-dimensional modelling analysis of the HST data for one object from each of the four sub-samples. The left-hand panels show cut-outs of the reduced HST WFPC2 images centred on the radio galaxies. The middle panels show the best-fitting host-galaxy models. The right-hand panels show the residual images produced by subtracting the best-fitting models from the reduced radio-galaxy images. All panels are $12.75^{\prime \prime} \times 12.75^{\prime \prime}$. 
and the full ZP5 sample can be found in Table 4. A graphical representation of the modelling process is shown in Fig 2 which, for one radio galaxy from each sub-sample, shows postage-stamp images of the reduced HST data, the bestfitting two-dimensional models and the residual images after subtraction of the best-fitting models.

\subsection{Morphology}

The mean value of the Sérsic $\beta$ parameter for the full ZP5 sample is $\langle\beta\rangle=0.23 \pm 0.01$, consistent with that expected for classical elliptical galaxies (the typical uncertainty in $\beta$ is \pm 0.05$)$. The average $\beta$ values displayed in Table 4 for the four radio-galaxy sub-samples show no evidence for any trend between the $\beta$ parameter and radio luminosity.

In Fig 3 we show a histogram of the derived $\beta$ parameters for the full ZP5 sample. Although Fig 3 confirms that the morphologies of the ZP5 sample radio galaxies are consistent with the classical $\beta=0.25$ value, we note here that there are a substantial number of objects with $\beta<0.25$. The possible implications of this in light of the recent discovery of a correlation between $\beta$ and central black-hole mass (Graham et al. 2001; Erwin et al. 2003) will be discussed in Section 8.1.

The distribution of $\beta$ parameters derived for the ZP5 sample is in good agreement with that determined by Dunlop et al. (2003) for their sample of twenty 3C-class radio galaxies and radio-loud quasars at $z \simeq 0.2$. The mean $\beta$ parameter determined by Dunlop et al. for their radio-loud AGN sample was $\langle\beta>=0.24 \pm 0.01$. Unfortunately there are no comparable samples in the literature of radio galaxies at higher redshift, for which Sérsic $\beta$ parameters have been derived, with which to compare the morphological results derived for the present sample.

\subsubsection{Model-subtracted images}

In the final column of Table 3 brief comments are listed describing the structure revealed by subtracting the bestfitting axially-symmetric host-galaxy model from the radiogalaxy images (see Fig 2 for examples). Objects for which the best-fitting galaxy model produces a clean subtraction, with little or no residual structure present in the subtracted images, are described as being undisturbed (UD). These objects comprise one third of the full ZP5 sample. Objects which show clear signs of interaction are described as being mergers (M), with those cases where the secondary nucleus/companion object has a luminosity $>10 \%$ of that of the radio-galaxy being described as major mergers (MM). Objects for which the modelling indicates the presence of an unresolved point-source nucleus with luminosity $>10 \%$ of that of the radio galaxy (eg. TOOT $1303+3334$, 7C $1731+6638)$ are described as possible quasar cores (QC?). Finally, those objects whose modelsubtracted images reveal symmetric, central positive flux residuals are described as having possible disc or aligned emission (DA?).

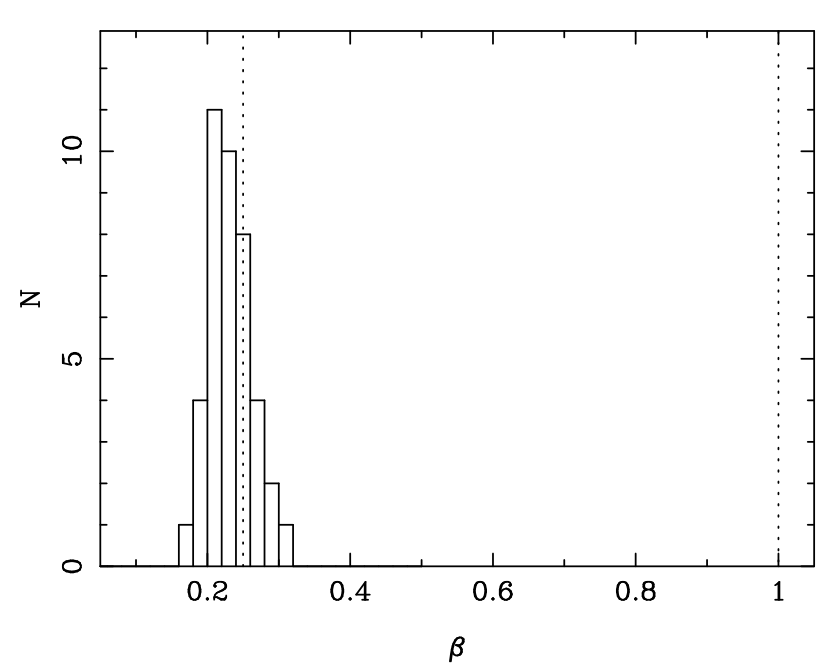

Figure 3. Histogram of the derived Sérsic $\beta$ parameters for the full ZP5 sample. The dotted vertical lines indicate the locations of $\beta=0.25$ and $\beta=1.0$, the expected values for classic de Vaucouleurs $r^{1 / 4}$ and Freeman exponential disk profiles respectively (de Vaucouleurs 1953; Freeman 1970).

The information provided by the model-subtracted images is not pursued further within this paper, which concentrates on the properties of the dominant smooth stellar component of the radio-galaxy hosts. However, this information will be fully exploited by a forthcoming paper which explores the link between radio luminosity/structure and the kpc-scale environments of the radio galaxies, the incidence and magnitude of interaction/merger activity, aligned emission components and the properties of those radio galaxies with possible quasar cores (Mitchell et al., in prep).

\subsection{Host-galaxy luminosity}

The absolute magnitudes of the radio galaxies have been calculated under the assumption that the spectral energy distributions of the hosts can be well represented by the passively evolving stellar populations typical of massive early-type galaxies. This decision is based on several lines of evidence, including the morphological results presented in the previous section, the $R-K$ colour versus redshift distribution of 7CRS objects (Willott, Rawlings \& Blundell 2001) and the off-nuclear spectroscopy of $z \simeq 0.2$ quasar host galaxies (Nolan et al. 2000). Furthermore, the initial results from our follow-up spectroscopic observations, designed to determine the host-galaxy stellar-velocity dispersions, indicate that the host-galaxy spectra are consistent with mature stellar populations (Willott et al., in prep).

The absolute magnitudes for each host galaxy presented in Table 3 are based on the integrated instrumental (F785LP) magnitudes returned by the modelling code. These instrumental magnitudes are then converted to apparent $I$-Cousins magnitudes using the filter transformations and colour equations detailed in Holtzman et al. (1995). The apparent colours required for this transformation were calculated from the 1995 version of Bruzual \& Charlot singleburst elliptical galaxy stellar-population models (Bruzual \& 
Table 3. Host-galaxy parameters derived from the two-dimensional modelling of the HST WFPC2 data. Column one lists the radiogalaxy names. Column 2 lists the effective radii in kpc. Column 3 lists the mean $R$-band surface brightness within the effective radii in mag. $\operatorname{arcsec}^{-2}$. The surface brightnesses have been corrected for $(1+z)^{4}$ cosmological dimming, and then K-corrected and corrected for passive evolution as described in the text. Column four lists the axial ratios and column five lists the real optical position angles of the radio galaxies in degrees, transformed from the fitted on-chip position angles. Column six lists the Sérsic $\beta$ parameters. Column 7 lists the integrated absolute $R$-band host luminosities, including K-correction and correction for passive evolution as described in the text. Column 8 lists the host-galaxy luminosities in units of $L^{\star}$, where $L^{\star}$ is taken to be $M_{R}=-22.07$ (Yagi et al. 2002). Column 9 lists the ratio of apparent nuclear:host luminosity. Column 10 lists the estimated black-hole mass for each object based on its fitted bulge luminosity as described in Section 8. Column 11 highlights properties of the radio galaxies revealed by the model-subtracted images: $\mathrm{UD}=$ undisturbed, $\mathrm{M}=$ merger, $\mathrm{MM}=$ major merger, DA?=possible disc/aligned emission, QC?=possible quasar core (see text for discussion).

\begin{tabular}{|c|c|c|c|c|c|c|c|c|c|c|}
\hline Source & $r_{e}$ & $<\mu>_{e}$ & $a / b$ & $\mathrm{PA}$ & $\beta$ & $M_{R}$ & $L / L^{\star}$ & $L_{n u c} / L_{\text {host }}$ & $\log \left(M_{b h} / \mathrm{M}_{\odot}\right)$ & Comments \\
\hline $3 \mathrm{C} 16$ & 22.9 & 21.75 & 1.43 & 152 & 0.20 & -23.23 & 2.9 & 0.028 & 8.87 & DA? \\
\hline 3C 19 & 30.9 & 21.86 & 1.31 & 55 & 0.21 & -23.81 & 5.0 & 0.019 & 9.17 & $\mathrm{M} / \mathrm{DA} ?$ \\
\hline $3 \mathrm{C} 46$ & 15.8 & 20.78 & 1.32 & 129 & 0.21 & -23.49 & 3.7 & 0.002 & 9.00 & MM \\
\hline $3 \mathrm{C} 172$ & 12.6 & 20.65 & 1.18 & 4 & 0.23 & -23.25 & 3.0 & 0.011 & 8.89 & UD \\
\hline $3 \mathrm{C} 200$ & 13.2 & 20.15 & 1.68 & 141 & 0.26 & -23.48 & 3.6 & 0.059 & 9.00 & DA? \\
\hline $3 \mathrm{C} 225 \mathrm{~B}$ & 18.6 & 21.04 & 1.42 & 115 & 0.23 & -23.49 & 3.7 & 0.025 & 9.00 & UD \\
\hline $3 \mathrm{C} 228$ & 13.2 & 21.17 & 1.38 & 105 & 0.25 & -22.64 & 1.7 & 0.113 & 8.58 & $\mathrm{M} / \mathrm{QC} ?$ \\
\hline $4 \mathrm{C} 74.16$ & 18.6 & 21.64 & 1.28 & 48 & 0.20 & -23.04 & 2.5 & 0.065 & 8.78 & DA? \\
\hline $3 \mathrm{C} 244.1$ & 15.8 & 20.76 & 1.40 & 82 & 0.25 & -23.45 & 3.5 & 0.060 & 8.98 & DA? \\
\hline 3C 274.1 & 9.5 & 20.20 & 1.09 & 116 & 0.26 & -23.18 & 2.8 & 0.000 & 8.85 & UD \\
\hline $3 \mathrm{C} 295$ & 29.5 & 21.03 & 1.44 & 136 & 0.29 & -24.52 & 9.5 & 0.000 & 9.52 & MM \\
\hline $3 \mathrm{C} 330$ & 20.9 & 21.26 & 1.21 & 21 & 0.20 & -23.71 & 4.6 & 0.081 & 9.11 & MM \\
\hline $3 \mathrm{C} 341$ & 16.6 & 21.08 & 1.16 & 115 & 0.20 & -23.45 & 3.5 & 0.069 & 8.99 & DA? \\
\hline 3C 427.1 & 18.2 & 20.68 & 1.42 & 31 & 0.25 & -23.81 & 5.0 & 0.000 & 9.16 & UD \\
\hline 3C 457 & 14.1 & 20.40 & 1.28 & 14 & 0.22 & -23.67 & 4.4 & 0.013 & 9.09 & DA? \\
\hline $6 \mathrm{C} 0825+3407$ & 14.5 & 21.12 & 1.09 & 112 & 0.27 & -23.14 & 2.7 & 0.000 & 8.83 & M \\
\hline $6 \mathrm{C} 0850+3747$ & 12.6 & 20.41 & 1.34 & 160 & 0.24 & -23.35 & 3.2 & 0.131 & 8.93 & UD/QC? \\
\hline $6 \mathrm{C} 0857+3945$ & 11.7 & 20.15 & 1.34 & 6 & 0.22 & -23.42 & 3.5 & 0.011 & 8.97 & UD \\
\hline 6C $1111+3940$ & 5.2 & 19.63 & 1.06 & 174 & 0.25 & -22.48 & 1.4 & 0.290 & 8.65 & $\mathrm{UD} / \mathrm{QC} ?$ \\
\hline 6C $1132+3439$ & 10.5 & 20.40 & 1.35 & 10 & 0.22 & -22.96 & 2.3 & 0.028 & 8.74 & UD \\
\hline $6 \mathrm{C} 1200+3416$ & 20.0 & 21.55 & 1.15 & 50 & 0.17 & -23.35 & 3.2 & 0.000 & 8.93 & MM \\
\hline $6 \mathrm{C} 1303+3756$ & 12.3 & 20.65 & 1.09 & 106 & 0.20 & -23.28 & 3.1 & 0.067 & 8.90 & MM/DA? \\
\hline $7 \mathrm{C} 0213+3418$ & 6.3 & 19.47 & 1.09 & 69 & 0.24 & -22.99 & 2.3 & 0.000 & 8.75 & UD \\
\hline $7 \mathrm{C} 0219+3419$ & 10.9 & 20.45 & 1.53 & 124 & 0.26 & -22.87 & 2.1 & 0.042 & 8.69 & UD \\
\hline $7 \mathrm{C} 0219+3423$ & 7.8 & 20.67 & 1.08 & 132 & 0.25 & -22.26 & 1.2 & 0.031 & 8.39 & UD \\
\hline $7 \mathrm{C} 0220+2952$ & 12.9 & 21.58 & 1.09 & 87 & 0.24 & -22.43 & 1.4 & 0.023 & 8.47 & UD \\
\hline $7 \mathrm{C} 0223+3415$ & 9.8 & 20.51 & 1.42 & 139 & 0.22 & -22.62 & 1.7 & 0.047 & 8.57 & UD \\
\hline $7 \mathrm{C} 0810+2650$ & 14.8 & 21.16 & 1.05 & 62 & 0.23 & -23.22 & 2.9 & 0.080 & 8.87 & UD \\
\hline $7 \mathrm{C} 1731+6638$ & 6.0 & 20.47 & 1.36 & 48 & 0.22 & -21.67 & 0.7 & 0.268 & 8.10 & $\mathrm{UD} / \mathrm{QC} ?$ \\
\hline 7C $1806+6831$ & 14.8 & 20.54 & 1.21 & 148 & 0.19 & -23.68 & 4.4 & 0.000 & 9.10 & M \\
\hline TOOT $0009+3523$ & 12.6 & 20.48 & 1.97 & 86 & 0.29 & -22.87 & 2.1 & 0.037 & 8.70 & $\mathrm{MM}$ \\
\hline TOOT $0013+3459$ & 15.5 & 19.85 & 1.64 & 128 & 0.30 & -24.14 & 6.8 & 0.000 & 9.33 & M \\
\hline TOOT $0018+3510$ & 19.5 & 21.21 & 1.32 & 49 & 0.20 & -23.51 & 3.8 & 0.039 & 9.01 & DA? \\
\hline TOOT $1255+3556$ & 7.8 & 20.42 & 1.07 & 2 & 0.25 & -22.53 & 1.5 & 0.155 & 8.52 & $\mathrm{UD} / \mathrm{QC} ?$ \\
\hline TOOT $1301+3658$ & 21.9 & 21.17 & 1.29 & 140 & 0.20 & -23.79 & 4.9 & 0.076 & 9.16 & DA? \\
\hline TOOT $1303+3334$ & 5.1 & 19.69 & 1.26 & 16 & 0.21 & -22.15 & 1.1 & 0.743 & 8.42 & $\mathrm{DA} ? / \mathrm{QC} ?$ \\
\hline TOOT $1307+3639$ & 12.0 & 20.31 & 1.12 & 110 & 0.22 & -23.51 & 3.8 & 0.084 & 9.01 & DA? \\
\hline TOOT $1309+3359$ & 6.6 & 19.84 & 1.11 & 56 & 0.21 & -22.70 & 1.8 & 0.025 & 8.61 & DA? \\
\hline TOOT $1626+4523$ & 7.4 & 19.54 & 1.19 & 70 & 0.23 & -23.19 & 2.8 & 0.060 & 8.86 & DA? \\
\hline TOOT $1630+4534$ & 10.2 & 19.71 & 1.03 & 126 & 0.23 & -23.88 & 5.2 & 0.028 & 9.20 & MM \\
\hline TOOT $1648+5040$ & 9.5 & 19.71 & 2.09 & 1 & 0.27 & -22.97 & 2.3 & 0.000 & 8.75 & DA? \\
\hline
\end{tabular}

Charlot 1993), under the assumption of a $z=5.0$ formation redshift. Following correction for cosmological dimming within our chosen cosmology, evolution and $K$-corrections were then applied using the same Bruzual \& Charlot models. Galactic extinction corrections were then applied to each object (Schlegel et al. 1998), before a final conversion to absolute $R$-Cousins magnitudes was made, assuming a $z=0$ colour of $R-I=0.7$ (Bruzual \& Charlot 1993), in order to facilitate a straightforward comparison with literature results on radio-galaxy properties. The surface-brightness results presented in Tables $3 \& 4$ have been corrected for $(1+z)^{4}$ cosmological dimming, and then K-corrected and corrected for passive evolution in the same fashion as the absolute magnitudes. The host-galaxy luminosities presented in Tables $3 \& 4$ and Fig 4 demonstrate that the host galaxies of all four sub-samples are luminous, with a mean inte- 
Table 4. Average values of the host-galaxy parameters derived from the two-dimensional modelling for the four radio-galaxy sub-samples and the ZP5 sample as a whole. For each parameter the mean value for the relevant sample is listed with its associated standard error, and the median value is listed immediately below. Column 2 lists the Sérsic $\beta$ parameters. Column 3 lists the axial ratios, while column 4 lists the effective scalelengths in kpc. Column 5 lists the mean $R$-band surface brightness within the effective radius in mag.arcsec ${ }^{-2}$. Column 6 lists the absolute integrated $R$-band magnitudes, while column 7 lists the host-galaxy luminosities in units of $L^{\star}$.

\begin{tabular}{lllllll}
\hline Sample & \multicolumn{1}{c}{$\beta$} & \multicolumn{1}{c}{$a / b$} & \multicolumn{1}{c}{$r_{e}$} & \multicolumn{1}{c}{$\langle\mu\rangle_{e}$} & \multicolumn{1}{c}{$M_{R}$} & \multicolumn{1}{c}{$L / L_{\star}$} \\
\hline 3CRR & $0.23 \pm 0.01$ & $1.33 \pm 0.04$ & $18.1 \pm 1.5$ & $20.96 \pm 0.14$ & $-23.48 \pm 0.11$ & $3.95 \pm 0.46$ \\
& 0.23 & 1.32 & 16.8 & 21.03 & -23.48 & 3.65 \\
\hline $6 \mathrm{CE}$ & $0.23 \pm 0.01$ & $1.20 \pm 0.05$ & $12.4 \pm 1.6$ & $20.56 \pm 0.24$ & $-23.14 \pm 0.12$ & $2.78 \pm 0.27$ \\
& 0.23 & 1.15 & 12.3 & 20.41 & -23.28 & 3.06 \\
\hline 7CRS & $0.23 \pm 0.01$ & $1.23 \pm 0.07$ & $10.4 \pm 1.3$ & $20.61 \pm 0.22$ & $-22.72 \pm 0.22$ & $2.08 \pm 0.41$ \\
& 0.24 & 1.15 & 9.4 & 20.53 & -22.74 & 1.87 \\
\hline TOOT & $0.25 \pm 0.01$ & $1.37 \pm 0.11$ & $11.7 \pm 1.6$ & $20.18 \pm 0.18$ & $-23.20 \pm 0.19$ & $3.28 \pm 0.54$ \\
& 0.23 & 1.26 & 10.3 & 19.85 & -23.19 & 2.82 \\
\hline FULL & $0.23 \pm 0.01$ & $1.30 \pm 0.04$ & $13.9 \pm 0.9$ & $20.61 \pm 0.10$ & $-23.20 \pm 0.09$ & $3.21 \pm 0.26$ \\
& 0.23 & 1.28 & 12.8 & 20.54 & -23.25 & 2.97 \\
\hline
\end{tabular}

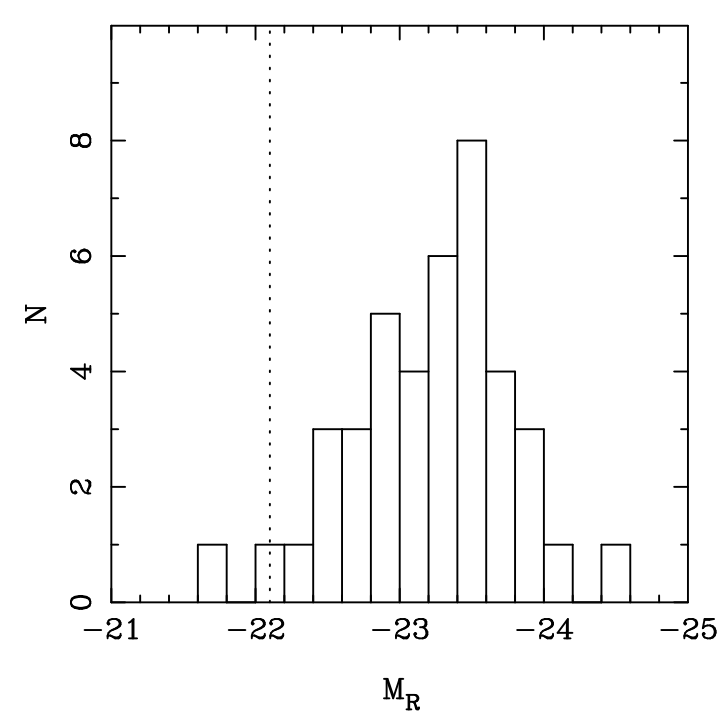

Figure 4. Histogram of integrated absolute $R$-band host magnitudes for the full ZP5 sample. The vertical dotted line indicates the location of $L^{\star}$ for local cluster galaxies: $M_{R}=-22.07$ (Yagi et al. 2002).

grated $R$-band absolute magnitude for the full ZP5 sample of $\left\langle M_{R}\right\rangle=-23.20 \pm 0.09$. In fact, these luminosities are directly comparable to the luminosities of galaxies drawn from the bright end of the local cluster-galaxy luminosity function. A comparison with the recent study of Yagi et al. (2002) shows that the mean host-galaxy luminosity of the full ZP5 sample is $3.2 \pm 0.3 L^{\star}$, where the Yagi et al. determination of $L^{\star}$ for local cluster galaxies is $M_{R}=-22.07$ in our adopted cosmology.

The mean host-galaxy luminosities presented in Table 4 also allow a comparison to be made with the host-galaxy luminosity differences between the $3 \mathrm{C}, 6 \mathrm{C}$ and $7 \mathrm{C}$ samples determined from the $K-z$ diagram (Willott et al. 2003; Eales et al. 1997). In the $K$-band, Willott et al. find a mean luminosity difference of 0.33 magnitudes between the $3 \mathrm{C}$ and $6 \mathrm{C}$ samples, and a corresponding difference of 0.55 magnitudes between the $3 \mathrm{C}$ and $7 \mathrm{C}$ samples. The equivalent figures from the analysis of our HST imaging are $0.34 \pm 0.16$ magnitudes and $0.76 \pm 0.25$ magnitudes in the $R$-band, in good agreement with the $K$-band results.

The rough scaling between extended radio luminosity and host-galaxy luminosity apparent from both the $K-z$ relation and the new results presented here, suggests a correlation exists between radio luminosity and black-hole mass within the $3 \mathrm{CRR}, 6 \mathrm{CE}$ and $7 \mathrm{CRS}$ samples. This issue is discussed further in Section 9.

\section{$5.3 \quad$ Host-galaxy scalelengths}

The best-fitting scalelength from the two-dimensional modelling of each individual member of the ZP5 sample is listed in Table 3, while the mean and median values for the four radio-galaxy sub-samples and the full ZP5 sample are listed in Table 4.

The full range of scalelengths displayed by the objects in the ZP5 sample is $5<r_{e}<31 \mathrm{kpc}$, with a mean value of $13.9 \pm 0.9 \mathrm{kpc}$. Scalelengths of this size clearly place the radio galaxies in the régime of brightest cluster galaxies (eg. Graham et al. 1996; Hoessel, Oergerle \& Schneider 1987) and a comparison of the ZP5 host galaxies with the properties of brightest cluster galaxies (BCGs) is pursued further in Section 7. Furthermore, in Section 6 the scalelength results for the 3CRR sub-sample are combined with existing literature results on 3C-class galaxies to search for any evidence for dynamical evolution amongst the most powerful radio galaxies within the redshift interval $0.0<z<0.8$.

In common with the results regarding the ZP5 hostgalaxy luminosities, an inspection of Table 4 reveals an apparent correlation between mean scalelength and radio luminosity within the $3 \mathrm{CRR}, 6 \mathrm{CE}$ and $7 \mathrm{CRS}$ sub-samples. This is entirely as expected given the correlation between hostgalaxy and radio luminosity discussed above, and the well known correlation between host luminosity and scalelength among early-type galaxies (e.g. $r_{e} \propto L_{\text {host }}^{0.63 \pm 0.03}$; Bernardi et al. 2003) 

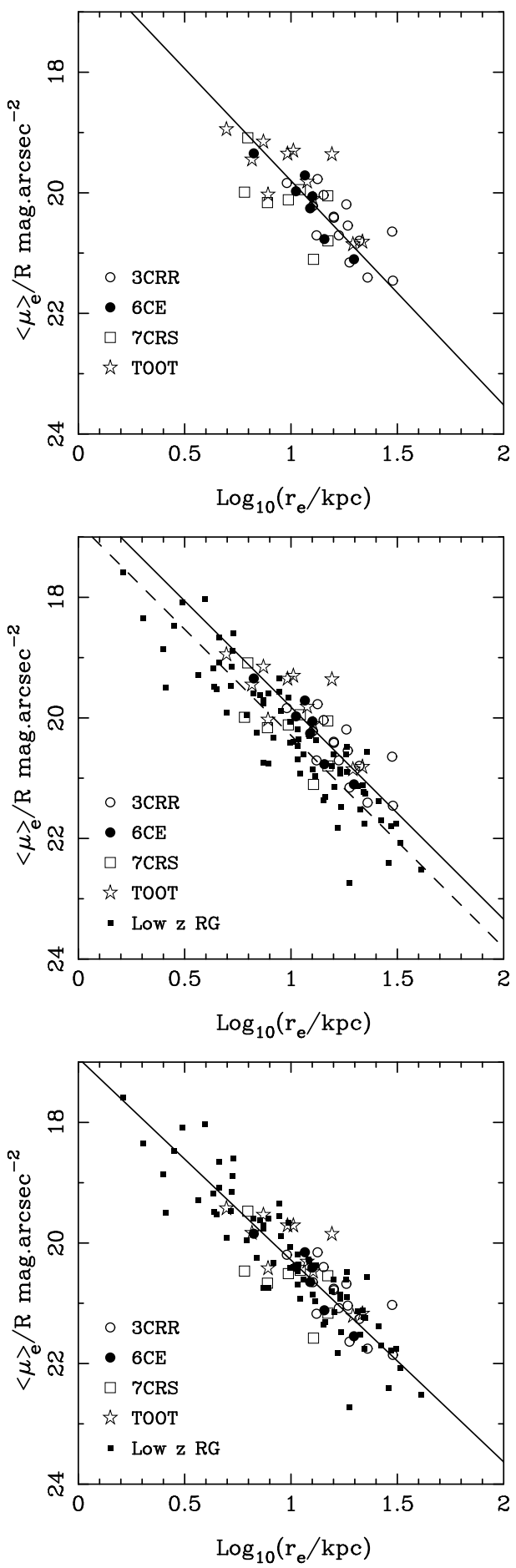

Figure 5. The top panel shows the best-fitting Kormendy relation for the full ZP5 sample, before the application of any evolutionary corrections. The middle panel shows the same ZP5 data with the addition of the 73 low-redshift radio galaxies from Bettoni et al. (2001). The best-fitting fixed-slope Kormendy relations to the ZP5 and Bettoni et al. radio galaxies are shown as the solid and dashed lines respectively (see text for discussion). The bottom panel shows the best-fitting Kormendy relation to the combined sample of the ZP5 and Bettoni et al. radio galaxies. In the bottom panel the ZP5 host galaxies have been corrected for passive evolution of their stellar populations as described in the text.

\subsection{The Kormendy relation}

Early-type galaxies are known to exist on a two-dimensional manifold (fundamental plane) in the three-dimensional parameter space defined by effective scalelength, the mean surface brightness within the effective scalelength and the central stellar-velocity dispersion (eg. Dressler, Lynden-Bell \& Burstein 1987; Djorgovski \& Davis 1987). At present we are engaged in obtaining stellar-velocity dispersion measurements for the ZP5 sample to investigate their location on the fundamental plane, and to estimate the masses of their central black holes via the $M_{b h}-\sigma$ correlation (Willott et al., in prep). However, armed with just the scalelength and surface brightness parameters for each host galaxy, it is possible to examine the photometric projection of the full fundamental plane, the so-called Kormendy or $\mu_{e}-r_{e}$ relation (Kormendy 1977).

The Kormendy relation for the ZP5 sample is shown in the top panel of Fig 5 . In this panel the $\langle\mu\rangle_{e}$ values of the ZP5 objects have been K-corrected and corrected for $(1+z)^{4}$ surface-brightness dimming, but have not been corrected for passive evolution. Using the iterative $\chi^{2}$ FITEXY routine, which takes account of errors in both parameters (Press et al. 1992), the best-fitting form of the Kormendy relation for the non-evolutionary corrected ZP5 sample is:

$<\mu>_{e}=3.72( \pm 0.51) \log r_{e}+16.08( \pm 0.59)$

In the middle panel of Fig 5 the same ZP5 data are repeated, along with the 73 low-redshift radio galaxies from the fundamental plane study of Bettoni et al. (2001). The best-fitting relation to Bettoni et al. sample is:

$<\mu>_{e}=3.32( \pm 0.14) \log r_{e}+16.97( \pm 0.15)$

Given that the Bettoni et al. sample is virtually at redshift zero, the vertical offset between the Kormendy relation of the Bettoni et al. sample and that of the ZP5 sample is a direct indication of the amount of luminosity evolution between $z=0$ and $z=0.5$. If the two samples are fitted with a Kormendy relation of fixed slope 3.52 (intermediate to their two independent fits) then the best-fitting intercepts are $16.30( \pm 0.07)$ and $16.77( \pm 0.05)$, for the ZP5 and Bettoni et al. samples respectively. This directly implies $0.47 \pm 0.09$ magnitudes of $R$-band luminosity evolution between $\mathrm{z}=0.5$ and $z=0$. This is in excellent agreement with the evolutionary corrections derived from the $z_{\text {for }}=5$ Bruzual \& Charlot model, which predicts a mean value of $0.42 \pm 0.01$ magnitudes of passive luminosity evolution for the ZP5 sample.

In the bottom panel of Fig 5 the low-redshift radio galaxies of Bettoni et al. are again plotted, together with the now evolutionary corrected ZP5 objects. The solid line shows the best-fitting relation to the combined sample which has the form:

$<\mu>_{e}=3.35( \pm 0.13) \log r_{e}+16.93( \pm 0.14)$

which is in excellent agreement with the recent determination of the Kormendy relation slope $(3.33 \pm 0.09)$ for 9000 $z \simeq 0$ early-type galaxies drawn from the Sloan Digital Sky Survey (SDSS) by Bernardi et al. (2003). 

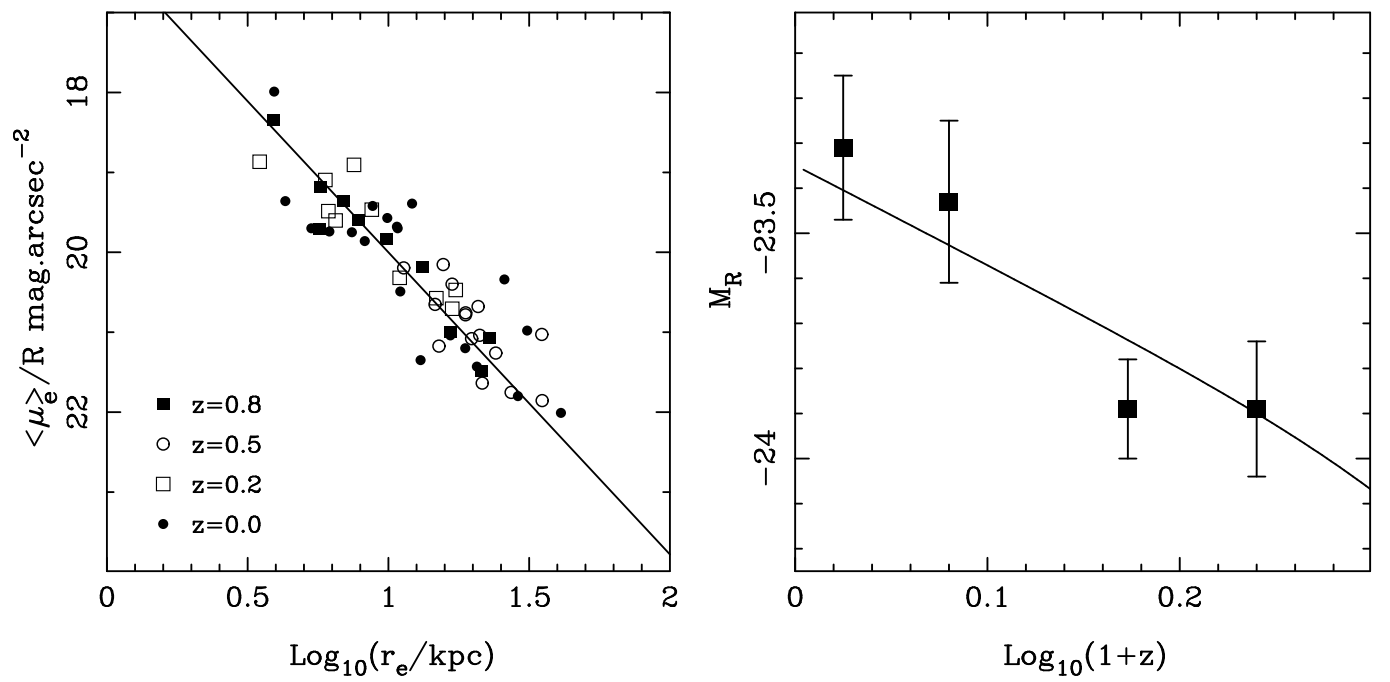

Figure 6. Panel A shows the Kormendy relation for the four 3C-class radio-galaxy samples described in the text. The surface brightnesses have been K-corrected and corrected for passive evolution as described in Section 5.2. The solid line is the best-fitting relation to the combined sample and has a slope of $3.78 \pm 0.30$. Panel B shows the evolution of the mean absolute $R$-band magnitudes (with standard error bars) of the four $3 \mathrm{C}$-class samples with redshift, where the absolute magnitudes have been K-corrected, but have not been corrected for passive evolution. The solid line shows the expected evolution of the mean absolute magnitude with redshift due to the passive evolution of their stellar populations, using the Bruzual \& Charlot single burst elliptical models with $z_{f o r}=5$ and assuming a redshift zero absolute magnitude of $M_{R}=-23.35$.

\section{EVOLUTION OF THE MOST POWERFUL RADIO GALAXIES}

In this section we focus on our new results for the $3 \mathrm{CRR}$ ZP5 sub-sample, combining them with previous work in the literature, to re-examine the issue of the evolution of $3 \mathrm{C}$ class radio galaxies from $z \simeq 0.8$ through to the present day. It has been known for some twenty years now that powerful radio galaxies follow a tight correlation between their apparent $K$-band magnitudes and redshift (Lilly \& Longair 1984). The simplest interpretation of this $K-z$ relation consistent with the data assumes that the radio galaxies were formed at high redshift $\left(z_{\text {for }}>3\right)$, either in a monolithic collapse or via hierarchical merging, and have been evolving passively thereafter.

However, this interpretation of the $K-z$ relation was challenged by Best, Longair \& Röttgering (1998) who argued that the apparent passive appearance of the $K-z$ relation was in fact a conspiracy. Based on evidence in the literature that points to higher redshift $3 \mathrm{C}$ radio galaxies $(z \geq 0.5)$ inhabiting significantly richer cluster environments (Hill \& Lilly 1991, Best 2000) than their low-redshift counterparts (eg. Prestage \& Peacock 1988; Lilly \& Prestage 1987), Best et al. proposed that $3 \mathrm{C}$ radio galaxies at $z \simeq 1$ and $z \simeq 0$ followed different evolutionary histories, and are seen as powerful radio sources at points within those histories at which they possessed similar stellar masses $\left(\simeq 10^{11} \mathrm{M}_{\odot}\right)$. Under this interpretation, higher redshift $3 \mathrm{C}$ galaxies would form first, via merger events within their rich cluster environments, reaching the required stellar mass by $z \simeq 1$, before evolving passively to form the radio-dormant brightest cluster galaxies (BCGs) seen at $z \simeq 0$. In contrast, the $3 \mathrm{C}$ radio galaxies at low redshift would reach the required stellar mass much later, via merging within their poorer cluster environments. The argument in favour of this interpretation of the
$K-z$ relation was further strengthened by Best et al.'s results suggesting that the scalelengths of $z \simeq 13 \mathrm{C}$ galaxies were significantly larger than seen in low-redshift 3C galaxies, and much more compatible with the characteristic sizes of low-redshift BCGs.

The evidence suggesting that $3 \mathrm{C}$ radio galaxies at $z \simeq 1$ have significantly larger scalelengths than typical of lowredshift $3 \mathrm{C}$ galaxies was re-examined using a full twodimensional analysis of the Best et al. HST imaging data by McLure \& Dunlop (2000). Comparison of the results of this analysis with previous results based on HST imaging of $3 \mathrm{C}$-class radio galaxies at $z \simeq 0.2$ (Dunlop et al. 2003; McLure et al. 1999) indicated that 3C galaxies at both redshifts had very similar characteristic scalelengths $(11 \pm 2$ kpc; $\left.H_{0}=50, \Omega_{0}=1\right)$. Here we can extend this comparison by including both the new results for the ZP5 3CRR subsample, and those for the twenty $3 \mathrm{C}$ galaxies included in the Bettoni et al. (2001) sample.

\subsection{Scalelengths}

The scalelength results for the $z \simeq 0$ sub-sample (Bettoni et al. 2001), and the $z \simeq 0.2$ and $z \simeq 0.8$ sub-samples (McLure $\&$ Dunlop 2000) were all derived by fitting classical $\beta=0.25$ de Vaucouleurs $r^{1 / 4}$ surface-brightness distributions. Consequently, in order to perform the scalelength comparison we also adopt the scalelength results for the ZP5 3CRR sub-sample obtained by restricting the fitting procedure to $\beta=0.25$.

Under this restriction the mean scalelength of the ZP5 $3 \mathrm{CRR}$ sub-sample is $16.3 \pm 1.6 \mathrm{kpc}$. When converted to our adopted cosmology the equivalent values for the $z \simeq 0, z \simeq$ 0.2 and $z \simeq 0.8$ samples are $14.2 \pm 2.2 \mathrm{kpc}, 9.8 \pm 1.6 \mathrm{kpc}$ and $11.4 \pm 2.2 \mathrm{kpc}$ respectively. In conclusion, these results 
show no indication of any trend for scalelength to vary with redshift in the interval $0.0<z<0.8$.

\subsection{Evolution of the Kormendy relation}

In panel A of Fig 6 we show the Kormendy relation for all four $3 \mathrm{C}$ radio-galaxy samples. The best-fitting relation to the combined sample is:

$<\mu>_{e}=3.78( \pm 0.30) \log r_{e}+16.22( \pm 0.33)$

where all four samples have been corrected for $(1+z)^{4}$ surface-brightness dimming, and have had both $\mathrm{K}$ corrections and evolutionary corrections applied as described in Section 5.2.

Panel A of Fig 6 demonstrates that within the redshift interval $0.0<z<0.8$ there is no obvious evidence for any dynamical evolution, as would be indicated by scalelength or surface-brightness evolution, other than that expected from purely passive evolution of the stellar populations. However, although we find no evidence for dynamical evolution of the class of AGN host galaxies which harbour powerful 3C-class radio sources in the redshift interval $0.0<z<0.8$, dynamical evolution of the individual host galaxies themselves within this redshift range cannot be ruled out. Panel B of Fig 6 shows that the redshift evolution of the mean absolute magnitudes of the four sub-samples is also consistent with purely passive evolution. However, it should also be pointed out that these results do not require that all $3 \mathrm{C}$ radio galaxies are formed in a monolithic collapse at high redshift, simply that the proto-galactic components which eventually form the host galaxies are formed reasonably co-evally, and that the vast majority of merger activity be completed at $z>1$. Furthermore, neither are the results presented in this section inconsistent with what would be expected under the $K-z$ interpretation proposed by Best et al. (1998).

A satisfactory discrimination between the two interpretations of the $K-z$ relation will rely on a consistent evaluation of the relative richness of the respective cluster environments at low and high redshift. Unfortunately, much of the work which has previously been done on the cluster environments of radio galaxies, particularly at low redshift, has relied on statistical determinations from single-filter imaging which are known to be subject to large uncertainties (eg. Barr et al. 2003). However, upon completion of our INT Wide Field Camera follow-up observations of the ZP5 sample, together with our existing observations of radio-galaxy samples at $z \simeq 0.2$ and $z \simeq 0.8$, we will be able to consistently investigate the evolution in the cluster environments of powerful radio galaxies, by using three-colour imaging to successfully separate true cluster members from foreground and background contaminants (Jarvis et al.; McLure et al., in prep).

\section{COMPARISON WITH BRIGHTEST CLUSTER GALAXIES}

The results regarding the scalelengths and integrated luminosities of the ZP5 sample presented in Section 5 suggest that the host galaxies of the ZP5 sample are directly comparable with brightest cluster galaxies (BCGs). This is in good agreement with the results of Dunlop et al. (2003) and

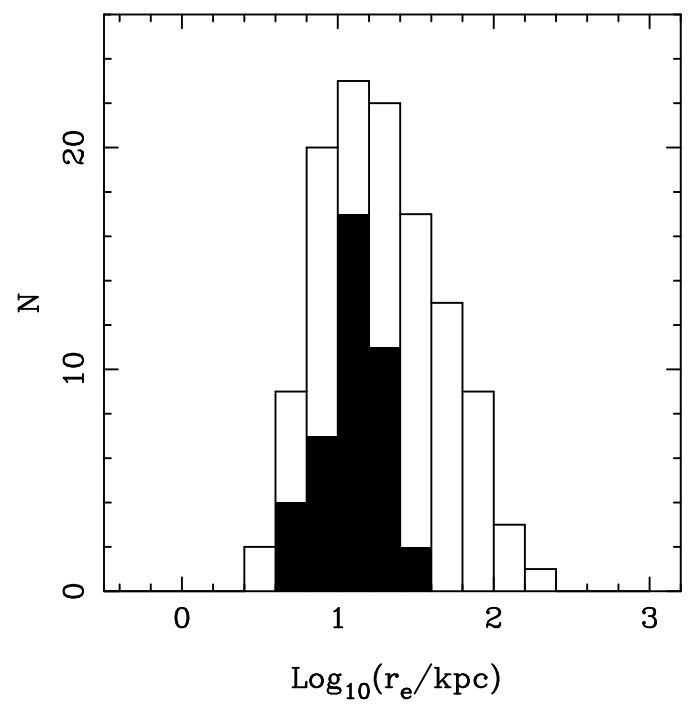

Figure 7. Scalelength histogram for the 119 Abell brightest cluster galaxies from Graham et al. (1996). Overlaid in black is the scalelength distribution of the full ZP5 sample.

McLure \& Dunlop (2001b) who concluded that the hosts of powerful radio-loud $\mathrm{AGN}$ at $z \simeq 0.2$ were directly comparable to the central BCGs of Abell class 0 clusters. In this section we explore this issue further via comparison with the findings of the HST imaging study of $z \simeq 0.5$ BCGs by Nelson et al. (2002), and the ground-based $R$-band surface photometry of 119 local Abell cluster BCGs by Graham et al. (1996).

Nelson et al. (2002) investigated the characteristic sizes of a heterogeneous sample of 16 BCGs, drawn from both $\mathrm{X}$-ray and optically selected cluster samples, using twodimensional modelling of HST NICMOS and WFPC2 imaging with the publically available GIM2D package (Simard 1998). The mean redshift of the Nelson et al. sample is $\langle z\rangle=0.54 \pm 0.03$, making it ideal for comparison with the results for the ZP5 sample. After conversion to our chosen cosmology, and correction for the effect of colour gradients (Nelson et al. 2002, Pahre 1999), we calculate the mean effective scalelength of the Nelson et al. BCGs to be $\left\langle r_{e}\right\rangle=$ $14.3 \pm 2.0 \mathrm{kpc}$, in excellent agreement with the scalelength distribution of the ZP5 sample; $\left\langle r_{e}\right\rangle=13.9 \pm 0.9 \mathrm{kpc}$. Given that the results for both the ZP5 and Nelson et al. samples are derived from similar two-dimensional modelling of HST imaging, of samples with identical redshift distributions, this can be taken as good evidence that the hosts of the ZP5 sample are indistinguishable from those of BCGs at $z \simeq 0.5$.

However, if the ZP5 scalelength results are compared to those determined for nearby Abell BCGs, the conclusions are more ambiguous. In Fig 7 we show a histogram of the effective scalelengths determined from one-dimensional $r^{1 / 4}$ fits to the $R$-band surface-brightness profiles of $119 z \simeq 0$ Abell cluster BCGs by Graham et al. (1996), together with the equivalent histogram for the ZP5 sample. The mean and median scalelengths determined by Graham et al. for the Abell BCGs are $28.7 \pm 2.7 \mathrm{kpc}$ and $18.5 \mathrm{kpc}$ respectively (after conversion to our adopted cosmology). The equivalent figures for the ZP5 sample are $13.9 \pm 0.9 \mathrm{kpc}$ and 12.8 kpc respectively. An application of the Kolmogorov-Smirnov 


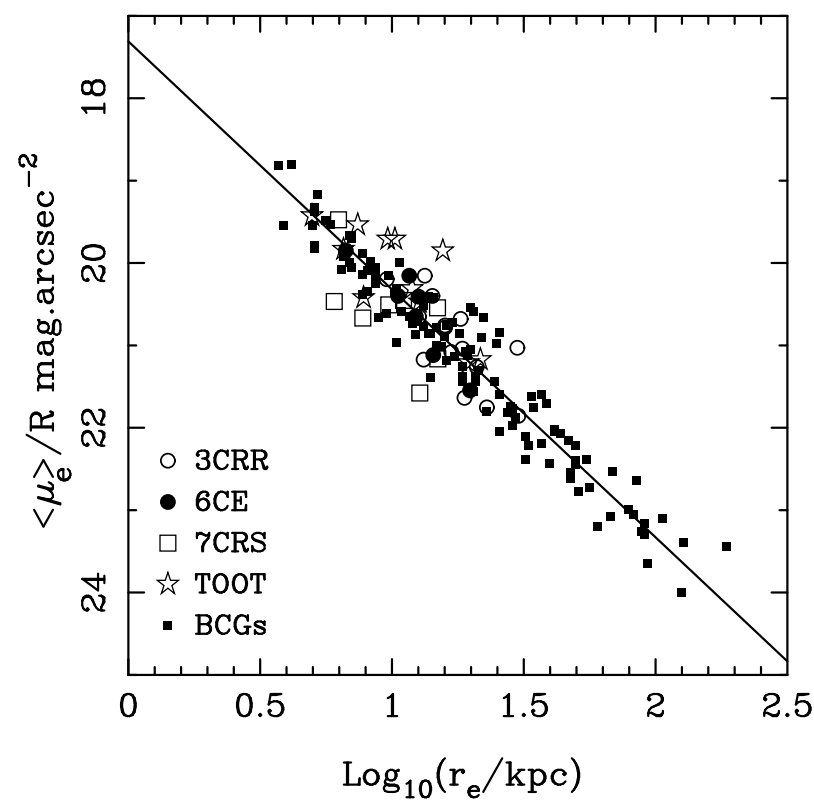

Figure 8. The combined Kormendy relation for the 119 Abell brightest cluster galaxies of Graham et al. (1996) and the full ZP5 sample. The solid line is the best-fitting relation to the combined sample and has a slope of $3.01 \pm 0.13$.

(KS) test finds the ZP5 and Abell cluster scalelength distributions to be drawn from different parent populations at high significance $\left(p=7 \times 10^{-4}\right)$

In Fig 8 the Kormendy relation for the ZP5 sample and the Abell BCGs is shown. It is clear from Fig 8 that the host galaxies of the ZP5 sample are fully consistent with the Kormendy relation for $z \simeq 0$ Abell BCGs, although the ZP5 sample, and $z \simeq 0.5$ BCGs in general (Nelson et al. 2002), appear to be devoid of galaxies with scalelengths $\gtrsim 40 \mathrm{kpc}$. However, the radio galaxies comprising the ZP5 sample are expected to inhabit a wide range of environments (Hill \& Lilly 1991), with previous results suggesting that the mean environmental richness should be comparable to low-redshift Abell class 0 clusters. Indeed, if the scalelength comparison with the Graham et al. Abell BCGs is restricted to only those objects in Abell class 0 clusters (67 of 119 objects) then the difference between the two distributions is only significant at the $2 \sigma$ level $(p=0.034)$, in agreement with Hill \& Lilly (1991) and McLure \& Dunlop (2001b).

In conclusion, the luminosities and scalelengths of the ZP5 sample show that the radio galaxies are consistent with the typical properties of BCGs at $z \simeq 0.5$, which are in turn comparable with the central galaxies of local Abell class 0 clusters. We note here that, as previously highlighted by Yates, Miller \& Peacock (1989), 3C295 is clearly an exceptional object in terms of it's cluster environment, which is known to be directly comparable to the richest clusters at low redshift (Hill \& Lilly 1991). Indeed, 3C295 is a factor of two more optically luminous than any other member of the 3CRR sub-sample and, as will be seen in Section 9, also inhabits an extreme region of the $L_{151 \mathrm{MHz}}-M_{b h}$ plane.

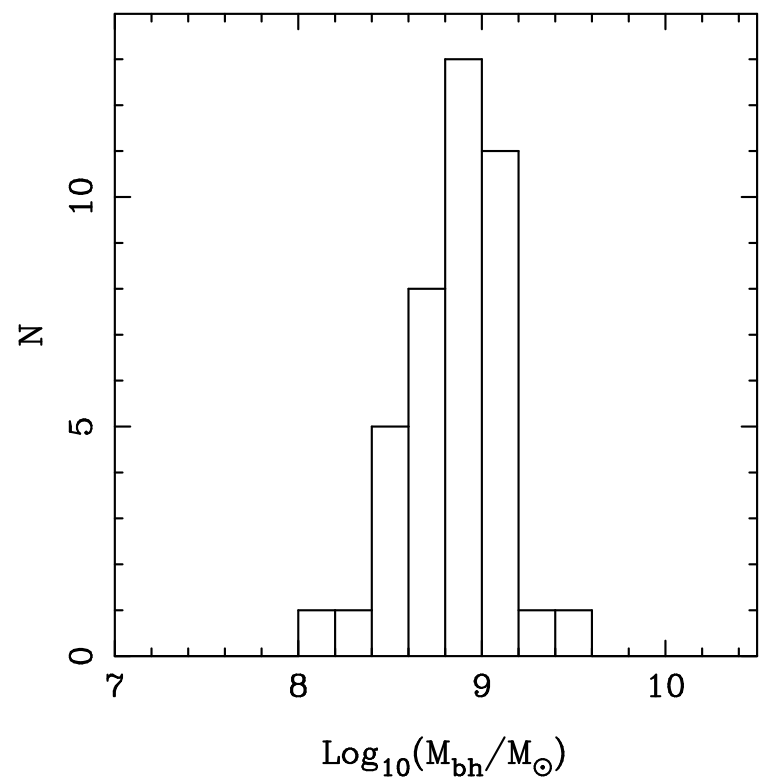

Figure 9. The black-hole mass distribution for the full ZP5 sample. The black-hole masses have been estimated from the fitted bulge luminosities via the $M_{b h}-M_{b u l g e}$ relation derived by McLure \& Dunlop (2002).

\section{THE BLACK-HOLE MASS DISTRIBUTION}

As discussed in Section 5, the morphological analysis of the HST imaging data clearly indicates that the ZP5 radio galaxies are close to being classical ellipticals. Furthermore, it is possible to directly link the hosts of the ZP5 sample with $z \simeq 0$ ellipticals via the application of passive evolutionary corrections. Consequently, under the assumption that, except for passive evolution, the $M_{b h}-M_{b u l g e}$ relation does not evolve between $z=0$ and $z=0.5$ we can proceed to estimate the central black-hole masses of the ZP5 sample using the correlation between black-hole mass and bulge luminosity observed at low redshift.

For this calculation we adopt the $M_{b h}-M_{\text {bulge }}$ relation as derived by McLure \& Dunlop (2002) for a combined sample of 72 AGN host galaxies and 18 low-redshift $(z<0.05)$ inactive ellipticals with black-hole mass measurements derived from either stellar or gas dynamics:

$\log M_{b h} / \mathrm{M}_{\odot}=-0.50( \pm 0.02) M_{R}-2.74( \pm 0.48)$

where $M_{R}$ is the absolute R-Cousins bulge luminosity (corrected to our adopted cosmology). It was demonstrated by McLure \& Dunlop (2002) that the scatter associated with this relation for elliptical galaxies is entirely consistent with that of the $M_{b h}-\sigma$ relation, at only 0.3 dex (see also Erwin et al. 2003). Recently, Marconi \& Hunt (2003) have performed detailed surface-brightness decompositions of 2-Micron All Sky Survey (2MASS) imaging of 37 low-redshift (distance $\lesssim$ $150 \mathrm{Mpc}$ ) galaxies with published black-hole mass determinations, investigating the level of scatter in the $M_{b h}-M_{b u l g e}$ relation in the near-infrared. Marconi \& Hunt confirm the McLure \& Dunlop (2002) results, extending them to demonstrate that the scatter associated with the $M_{b h}-M_{b u l g e}$ relation is $\simeq 0.3 \mathrm{dex}$, regardless of morphological type, provided that accurate disc/bulge decompositions of the galaxy surface-brightness distributions are adopted. The distribu- 
tion of black-hole masses for the full ZP5 sample as estimated from their bulge luminosities are shown in Fig 9.

The geometric mean black-hole mass for the ZP5 sample is $10^{8.87 \pm 0.04} \mathrm{M}_{\odot}$. This is in good agreement with the results of Bettoni et al. (2003) who determined a geometric mean black-hole mass of $10^{8.81 \pm 0.06} \mathrm{M}_{\odot}$ and $10^{8.91 \pm 0.06} \mathrm{M}_{\odot}$ for 45 low-redshift radio galaxies $(z \lesssim 0.1)$ using the $M_{b h}-\sigma$ and $M_{b h}-M_{b u l g e}$ relations respectively. When combined with the results of previous studies at lower redshift (eg. Laor 2000; McLure \& Dunlop 2002; McLure \& Jarvis 2002; Dunlop et al. 2003; Bettoni et al. 2003; Barth et al. 2003) it is clear that truly powerful radio-loud AGN, those capable of producing kpc-scale jets, are powered by black holes with masses greater than $10^{8} \mathrm{M}_{\odot}$. In addition, given that radio-loud AGN are drawn from the high-mass end of the black-hole mass function, the results of these studies suggest the existence of a maximum black-hole mass of $\simeq 3 \times 10^{9} \mathrm{M}_{\odot}$ (c.f. 3C295). This black-hole mass limit is in agreement with the maximum mass measured via gas or stellar dynamics in the local Universe (Ford et al. 1994; Tadhunter et al. 2003) and the limit implied from broad emission-line based blackhole mass estimates for $>12000 \operatorname{SDSS}$ quasars at $z \leq 2$ (McLure \& Dunlop 2004).

\subsection{On the correlation between $\beta$ and black-hole mass}

We note here that Graham et al. (2001) discovered a correlation between central black-hole mass and the Sérsic morphological parameter $\beta$ for a sample of 20 local galaxies with black-hole masses measured via gas or stellar dynamics. This correlation displays a comparable level of scatter $(\sim 0.3 \mathrm{dex})$ to that of the $M_{b h}-\sigma$ relation (eg. Tremaine et al. 2002). Due to the fact that black-hole mass in early-type galaxies also correlates with bulge luminosity with comparably low scatter (McLure \& Dunlop 2002; Marconi \& Hunt 2003), it is expected that a correlation will exist between the $\beta$ parameter and host-galaxy luminosity.

No such correlation is observed in the full ZP5 sample $\left(r_{s}=0.20, p=0.21\right)$. It is possible that the properties of two of the ZP5 sample, TOOT 0013+3459 and 3C295 (see Table 3), may have resulted in the determination of anomalously large $\beta$ parameters for their luminosities. However, following the exclusion of these two objects the correlation between $\beta$ and host luminosity is still only marginally significant $\left(r_{s}=0.40, p=0.012, \simeq 2.4 \sigma\right)$. The principal reason for this discrepancy may simply be that we have a much smaller dynamic range in $\beta$ compared to the Graham et al. (2001) study. However, we also note that the Graham et al. correlation between $\beta$ and black-hole mass would imply a mean black-hole mass for the ZP5 sample of $10^{8.4} \mathrm{M}_{\odot}$, for our determination of $\langle\beta\rangle=0.23 \pm 0.01$. This is significantly different to the figure of $10^{8.87 \pm 0.04} \mathrm{M}_{\odot}$ determined from the $M_{b h}-M_{b u l g e}$ relation which, using the Graham et al. $\beta-M_{b h}$ correlation, would imply $<\beta>=0.16$.

\section{THE BLACK HOLE MASS - RADIO LUMINOSITY CONNECTION}

The question of whether or not black-hole mass is correlated with radio emission in AGN has been the focus of a great deal of attention in the literature recently (eg. Dunlop et al. 2003; Lacy et al. 2001; Ho 2002; Woo \& Urry 2002). Using broad emission-line based virial black-hole mass estimates Lacy et al. (2001) investigated the radio luminosity - blackhole mass relationship for a combined sample composed of objects from the First Bright Quasar (FBQ) and PG quasar surveys. The results of this study showed a strong correlation between black-hole mass and $5-\mathrm{GHz}$ radio luminosity of the form $L_{5 G H z} \propto M_{b h}^{1.4 \pm 0.2}$ over a dynamic range of some 6 decades in radio luminosity and 4 decades in black-hole mass. By combining their black-hole mass estimates, derived from HST imaging of a sample of 33 AGN at $z \simeq 0.2$, with those of the Lacy et al. study, Dunlop \& McLure (2003) demonstrated that the $5-\mathrm{GHz}$ radio luminosity of both AGN and quiescent nearby galaxies appears to be confined between two radio-power limits. The lower radio-power limit defines the minimum radio luminosity that can be emitted by a given black-hole mass, and is well described by a relation of the form $L_{5 G H z} \propto M_{b h}^{2.5}$, in good agreement with the correlation originally observed for nearby quiescent galaxies by Franceschini, Vercellone \& Fabian (1998). The upper radio-power limit proposed by Dunlop \& McLure (2003) appears to be well described by a relation of the same functional form, offset from the lower limit by some 5 decades in radio luminosity. In contrast, the recent studies of Ho (2002) and Woo \& Urry (2002) find no convincing evidence for a correlation between black-hole mass and radio luminosity, in samples comprising a range in nuclear activity from local quiescent galaxies up to and including powerful quasars.

In this section we investigate whether there is a correlation between black-hole mass and radio luminosity within the ZP5 radio-galaxy sample. In contrast to previous studies, the majority of which have been based on high frequency (5-GHz) radio luminosity, the ZP5 radio-galaxy sample allows us to test for a correlation between black-hole mass and extended low-frequency $(151-\mathrm{MHz})$ radio luminosity. This distinction is potentially important given that $L_{151 \mathrm{M} \mathrm{Hz}}$ is less affected by beaming than $L_{5 G H z}$ (eg. Jarvis \& McLure 2002) and has a close relationship to the time-averaged kinetic energy of the jets (e.g. Rawlings \& Saunders 1991).

The results presented thus far are consistent with a picture in which extended low-frequency radio luminosity scales roughly with host-galaxy luminosity/mass. In combination with the latest determination of the $K-z$ relation (Willott et al. 2003) it appears that the most powerful 3C-class radio galaxies reside in galaxies with $R$-band luminosities of $\simeq 4 L^{\star}$, while the lower-luminosity $6 \mathrm{C}$ and $7 \mathrm{C}$-class radio galaxies typically inhabit hosts with luminosities of $\simeq 3 L^{\star}$ and $\simeq 2 L^{\star}$ respectively.

At present it is unclear how the TOOT sub-sample fits within this picture. It can be seen from the results presented in Table 4 that the TOOT galaxies do not appear to follow the rough scaling between extended radio luminosity and host-galaxy luminosity apparent in the 3CRR, 6CE and 7CRS sub-samples. Indeed, the mean luminosity of the TOOT sub-sample $\left(3.28 \pm 0.54 L^{\star}\right)$ is greater than that of both the 7CRS and 6CE sub-samples, and is consistent with the low-redshift results of Owen \& Laing (1989) that fat double/jet/FRI sources reside in hosts which are on average $\simeq 0.5$ magnitudes brighter than those of classical double/FRII sources of comparable radio luminosity. However, as was mentioned in Section 2, the TOOT sub-sample 

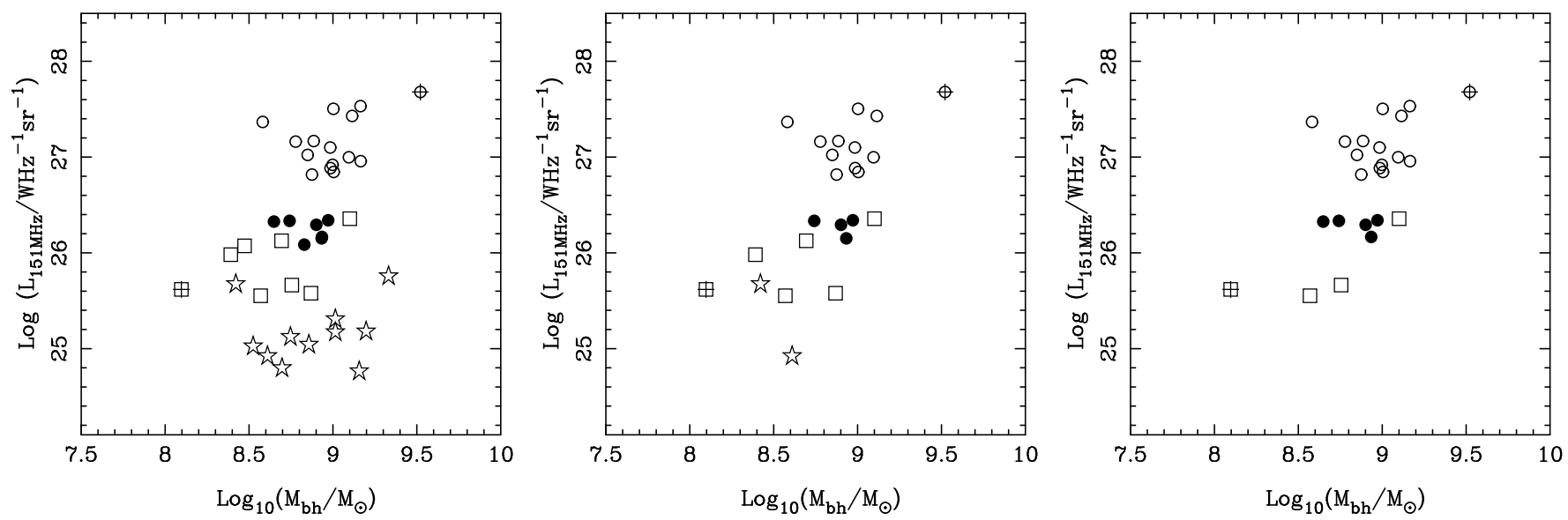

Figure 10. Panel A shows 151-MHz radio luminosity plotted against black-hole mass for the full ZP5 sample. Panel B shows the $L_{151}-M_{b h}$ plane for the ZP5 objects with HEG spectra. Panel C shows the $L_{151}-M_{b h}$ plane for the ZP5 objects with classical double (CD) radio structures. The black-hole masses have been estimated from the bulge luminosity fits using the $M_{b h}-M_{b u l g e}$ correlation of McLure \& Dunlop (2002). The two objects highlighted with crosses are 7CRS1731+6638 and 3C295 which possess the two extreme values of black-hole mass and have a tendency to lead the eye. However, these two objects are included in the correlation analysis unless stated otherwise.

was drawn from a preliminary version of the survey and it is unclear at the time of writing to what extent the current TOOT sub-sample is biased by the exclusion of the optically faintest sources.

With this in mind, panel A of Fig 10 shows $151-\mathrm{MHz}$ radio luminosity versus estimated black-hole mass for the full ZP5 sample, where the black-hole mass estimates have been derived via the McLure \& Dunlop (2002) $M_{b h}-M_{b u l g e}$ relation as described in Section 8. Taken as a whole, there is only a weak $\left(r_{s}=0.35, p=0.027,2.2 \sigma\right)$ correlation between black-hole mass and radio luminosity within the ZP5 sample. However, as suggested previously, it can be seen from panel A of Fig 10 that the apparent weakness of the $L_{151}-M_{b h}$ correlation displayed by the full ZP5 sample is due, at least in part, to the inclusion of the eleven TOOT objects.

To investigate the possible influence of radio structure and nuclear spectral type we have also plotted in Fig 10 the $L_{151}-M_{b h}$ relation for two sub-sets of the ZP5 sample. In panel B of Fig 10 we show the $L_{151}-M_{b h}$ relation for those objects which display high-excitation nuclear spectra (HEG) only. As is suggested by the figure, this subsample displays a fairly strong correlation which is significant at the $99.9 \%$ level $\left(r_{s}=0.62, p=0.001,3.0 \sigma\right)$, although this drops in significance to only $2.4 \sigma$ following the exclusion of $3 \mathrm{C} 295$ and 7CRS $1731+6638$ (objects highlighted with crosses in Fig 10). In panel C of Fig 10 we show the $L_{151}-M_{b h}$ relation for the ZP5 objects with classical double (CD) radio structures only, effectively excluding the TOOT sub-sample. This correlation is significant at the $2.7 \sigma$-level $\left(r_{s}=0.56, p=0.005\right)$, a result which is confirmed by the application of the generalized Kendall's tau test $(\tau=0.83$, $2.8 \sigma)$, although again we note that the significance drops to only $\simeq 2 \sigma\left(r_{s}=0.43, p=0.049\right)$ following the exclusion of $3 \mathrm{C} 295$ and 7CRS $1731+6638$.

It is clear from these results that $\mathrm{a} \simeq 3 \sigma$ correlation does exist between black-hole mass and low-frequency $151-\mathrm{MHz}$ radio luminosity within the $3 \mathrm{CRR}, 6 \mathrm{CE}$ and $7 \mathrm{CRS}$ samples, although the scatter in radio luminosity at a given black-hole mass is large $(\simeq 1.5 \mathrm{dex})$. This result is in good agreement with the Lacy et al. (2001) study of the $L_{5 G H z}-M_{b h}$ relation in broad-line quasars, a study which covered a much larger dynamic range in black-hole mass. Moreover, this result is also consistent with the hypothesis proposed by Dunlop \& McLure (2003) that the radio luminosity which can be produced by a given black-hole mass may be bounded by upper and lower limits separated by several orders of magnitude. Indeed, given that theoretical models indicate that the radio luminosity of AGN, in addition to black-hole mass, should also be related to both accretion rate and black-hole spin (eg. Meier 2003), it is perhaps unsurprising that the $L_{151}-M_{b h}$ correlation is not stronger.

Furthermore, at present it is unclear whether the objects which do not follow the $L_{151}-M_{b h}$ correlation, predominantly the lower-luminosity TOOT sub-sample, do so because of their LEG nature, their fat double/jet radio structures, or alternatively, as a result of a selection effect which biases the TOOT sub-sample towards the inclusion of objects with larger host luminosities/black-hole masses. A more detailed analysis of the connection between the radio properties and black-hole masses of the ZP5 sample will be pursued in a future paper (Mitchell et al., in prep).

\section{CONCLUSIONS}

The results of a detailed analysis of deep, high-resolution HST images of a sample of 41 radio galaxies which spans three decades in radio luminosity at $z \simeq 0.5$ have been presented. The host-galaxy properties derived from twodimensional image modelling have been discussed and compared to existing results in the literature. The principle conclusions of this study can be summarized as follows:

(i) The morphologies of the host galaxies of all four radiogalaxy sub-samples are found to be largely consistent with a canonical elliptical-galaxy $r^{1 / 4}$ surface-brightness distri- 
bution. No trend is detected for host-galaxy morphology to vary significantly with radio luminosity.

(ii) The luminosities of the host galaxies are found to be consistent with those of galaxies drawn from the bright end of the local cluster-galaxy luminosity function, in the range $0.7 L^{\star}<L<10 L^{\star}$, with a mean value of $3.2 \pm 0.3 L^{\star}$.

(iii) The radio-galaxy hosts are found to lie on the same Kormendy $\left(\mu_{e}-r_{e}\right)$ relation as powerful $z \simeq 0$ radio galaxies, after allowing for the effects of passive evolution of their stellar populations under the assumption of a high formation redshift $\left(z_{\text {for }} \gtrsim 3\right)$.

(iv) By combining the new results on $z \simeq 0.53 \mathrm{CRR}$ galaxies with previous results in the literature, it is found that there is no evidence for dynamical evolution among the hosts of 3C-class radio galaxies in terms of scalelength, luminosity and Kormendy relation in the redshift interval $0.0<z<0.8$. However, although no evidence is found for dynamical evolution among the hosts of the most powerful radio sources as a class, dynamical evolution of the individual host galaxies themselves cannot be ruled out.

(v) The scalelengths of the ZP5 host galaxies are found to be consistent with those of brightest cluster galaxies (BCGs) at the same cosmic epoch $(z \simeq 0.5)$. When compared to local Abell cluster BCGs the ZP5 host galaxies are found to be a factor of $\simeq 1.5$ smaller on average. However, when the comparison is restricted to local clusters of Abell class 0 only, the ZP5 and BCG scalelength distributions are found to be statistically indistinguishable.

(vi) Converting the derived host-galaxy luminosities into black-hole mass estimates using the local $M_{b h}-M_{b u l g e}$ relation predicts that the host galaxies harbour central blackholes in the mass range $10^{8.1} \mathrm{M}_{\odot}<M_{b h}<10^{9.5} \mathrm{M}_{\odot}$, with a geometric mean of $10^{8.87 \pm 0.04} \mathrm{M}_{\odot}$.

(vii) Significant correlations $(\simeq 3 \sigma)$ are found between black-hole mass and extended low-frequency radio luminosity for sub-samples of the ZP5 objects which have either classical double (CD) radio structures or high-excitation nuclear spectra (HEG). However, at present we are unable to determine whether the objects which do not follow the same correlation, predominantly the lower-luminosity TOOT subsample, do so because of their LEG spectra, fat double/jet radio structures, or alternatively, as a result of selection effects biasing the TOOT sub-sample towards the inclusion of objects with higher host luminosities/black-hole masses.

\section{ACKNOWLEDGMENTS}

RJM acknowledges the award of a PPARC postdoctoral fellowship and a PPARC PDRA. CJW thanks the National Research Council of Canada for support. MJJ acknowledges the support of the European Community Research and Training Network 'The Physics of the Intergalactic Medium' and a PPARC PDRA. This material is based in part upon work supported by the Texas Advanced Research Program under Grant No. 009658-0710-1999 and NASA/STScI grant HSTGO-09045.04-A. EM acknowledges the award of a PPARC studentship. JSD acknowledges the enhanced research time provided by the award of a PPARC Senior Fellowship. MW acknowledges the Swedish Research Council for travel funding to La Palma. Some of the data presented in this paper were obtained from the Multimission Archive at the Space
Telescope Science Institute (MAST). STScI is operated by the Association of Universities for Research in Astronomy, Inc., under NASA contract NAS5-26555. Support for MAST for non-HST data is provided by the NASA Office of Space Science via grant NAG5-7584 and by other grants and contracts. This research has made use of the NASA/IPAC Extragalactic Database (NED) which is operated by the Jet Propulsion Laboratory, California Institute of Technology, under contract with the National Aeronautics and Space Administration.

\section{REFERENCES}

Barr J.M., Bremer M.N., Baker J.C., Lehnert M.D., 2003, MNRAS, in press, astro-ph/0308128

Barth A.J., Ho L.C., Sargent W.L.W., 2003, ApJ, 583, 134

Best P.N., Longair M.S., Röttgering H.J.A., 1998, MNRAS, 295, 549

Best P.N., 2000, MNRAS, 317, 720

Bernardi M., et al., AJ, 2003, 125, 1849

Bettoni D., Falomo G., Fasano G., Govoni F., Salvo M., Scarpa R., 2001, A\&A, 380, 471

Bettoni D., Falomo G., Fasano G., Govoni F., 2003, A\&A, 399, 869

Bruzual G.A., Charlot S., 1993, ApJ, 405, 538

Butcher H., Oemler A., 1978, ApJ, 226, 559

de Vaucouleurs G., 1953, MNRAS, 113, 134

Djorgovski S., Davies M., 1987, ApJ, 313, 59

Dressler A., Lynden-Bell D., Burstein D., 1987, ApJ, 313, 42

Dunlop J.S., McLure R.J., 2003, in 'The Mass of Galaxies at Low and High Redshift'. Proceedings of the ESO Workshop held in Venice, Italy, 24-26 October 2001, p. 268.

Dunlop J.S., McLure R.J., Kukula M.J., Baum S.A., O’Dea C.P., Hughes D.H., 2003, MNRAS, 340, 1095

Eales S., Rawlings S., Law-Green D., Cotter G., Lacy M., 1997, MNRAS, 291, 593

Ellingson E., Yee H.K.C., Green R.F., 1991, ApJ, 378, 476

Erwin P., et al., 2003, Carnegie Observatories Astrophysics Series, Vol. 1: Coevolution of Black Holes and Galaxies, L. Ho Ed.

Ferrarese L., Merritt D., ApJ, 2000, 539, L9

Ford H.C., et al., 1994, ApJ, 435, L27

Franceschini A., Vercellone S., Fabian A.C., 1998, MNRAS, 297, 817

Freeman K.C., 1970, ApJ, 160, 811

Fruchter A.S., Hook R.N., 2002, PASP, 114, 144

Gebhardt K., et al., 2000, ApJ, 539, L13

Graham A., Lauer T.R., Colless M., Postman M., 1996, ApJ, 465, 534

Graham A., Erwin P., Caon N., Trujillo I., 2001, ApJ, 563, L11

Hill G.J., Lilly S.J., 1991, ApJ, 367, 1

Hill G., Rawlings S., 2003, NewAR, 47, 373

Ho L.C., 2002, ApJ, 564, 120

Hoessel J.G., Oegerle W.R., Schneider D.P., 1987, AJ, 94, 111

Holtzman J.A., Burrows C.J, Casertano S., Hester J.J., Trauger J.T., Watson A.M., Worthey G., 1995, PASP, 107, 1065

Jackson N., Rawlings S., 1997, MNRAS, 286, 241

Jarvis M.J., McLure R.J., 2002, MNRAS, 336, L38

Kaspi S., Smith P.S., Netzer H., Maoz D., Jannuzi B.T., Giveon U., 2000, ApJ, 533, 631

Kormendy J.G., 1977, ApJ, 217, 406

Kormendy J., Richstone D., 1995, ARA\&A, 33, 581

Krist J., TinyTim v6.0 User Manual, 2001

Lacy M., Laurent-Muehleisen S.A., Ridgway S.E., Becker R.H., White R.L., 2001, ApJ, 551, 17

Lacy M., Rawlings S., Hill G.J., Bunker A.J., Ridgway S.E., Stern D., 1999, MNRAS, 308, 1096 
Laor A., 2000, ApJ, 543, L111

Laing R.A., Riley J.M., Longair M.S., 1983, MNRAS, 204, 151

Lilly S.J., Longair M.S., 1984, MNRAS, 211, 833

Lilly S.J., Prestage R.M., 1987, MNRAS, 225, 531

Magorrian J., et al., 1998, AJ, 115, 2285

Marconi A., Hunt L.K., 2003, ApJ, 589, L21

McLure R.J., Kukula M.J., Dunlop J.S., Baum S.A., O’Dea C.P., Hughes D.H., 1999, MNRAS, 308, 377

McLure R.J., Dunlop J.S., 2000, MNRAS, 317, 249

McLure R.J., Dunlop J.S., Kukula M.J., 2000, MNRAS, 318, 693

McLure R.J., Dunlop J.S., 2001a, MNRAS, 327, 199

McLure R.J., Dunlop J.S., 2001b, MNRAS, 321, 515

McLure R.J., Dunlop J.S., 2002, MNRAS, 331, 795

McLure R.J., Jarvis M.J., 2002, MNRAS, 337, 109

McLure R.J., Dunlop J.S., 2004, MNRAS, submitted, astro$\mathrm{ph} / 0310267$

Meier D.L., 2003, NewAR, 47, 667

Nelson A.E., Simard L., Zaritsky D., Dalcanton J.J., Gonzalez A.H., 2002, ApJ, 567, 2002

Nolan L.A., Dunlop J.S., Kukula M.J., Hughes D.H., Boroson T., Jimenez R., 2000, MNRAS, 323, 308

Oegerle W.R., Hoessel J.G., 1991, ApJ, 375, 15

Owen F.N., Laing R.A., 1989, MNRAS, 238, 357

Pahre M.A., 1999, ApJS, 124, 127

Press W.H., Teukolsky S.A., Vetterling W.T., Flannery B.P., 1992, Numerical Recipes, Cambridge University Press

Prestage R.M., Peacock J.A., 1988, MNRAS, 230, 131

Rawlings S., Eales S., Lacy M., 2001, MNRAS, 322, 523

Rawlings S., Saunders R., 1991, Nature, 349, 138

Schlegel D.J., Finkbeiner D.P., Davis M., 1998, ApJ, 500, 525

Sérsic, J.L., 1968, Atlas de Galaxias Australes, Observatorio Astronómico de Córdoba, Argentina

Simard L., 1998, in ASP Conf. Ser. 145, Astronomical Data Analysis Software Systems VII, ed. Albrecht R., Hook R.N., Bushouse H.A., 108

Smith E.P., Heckman T.M., 1989, ApJ, 341, 658

Tadhunter C., Marconi A., Axon D., Wills K., Robinson T.G., Jackson N., 2003, MNRAS, 342, 861

Taylor G.T., Dunlop J.S., Hughes D.H., Robson E.I., 1996, MNRAS, 283, 930

Tremaine S., et al., 2002, ApJ, 574, 740

Vestergaard M., 2002, ApJ, 571, 733

Willott C.J., Rawlings S., Blundell K.M., Lacy M., 2000, MNRAS, 316, 449

Willott C.J., Rawlings S., Blundell K.M., 2001, MNRAS, 324, 1

Willott C.J., Rawlings S., Jarvis M.J.J., Blundell K.M., 2003, MNRAS, 339, 173

Wold M., Lacy M., Lilje P.B., Serjeant S., 2000, MNRAS, 316, 267

Wold M., Lacy M., Dahl H., Lilje P.B., Ridgway S.E., 2002, MNRAS, 335, 1017

Woo J., Urry C.M., 2002, ApJ, 579, 530

Yagi M, Kashikawa N., Sekiguchi M., Doi M., Yasuda N., Shimasaku K., Okamura S., 2002, AJ, 123, 87

Yates M.G., Miller L., Peacock J.A., 1989, MNRAS, 240, 129

Zirm A.W., Dickinson M., Dey A., 2003, ApJ, 585, 90 